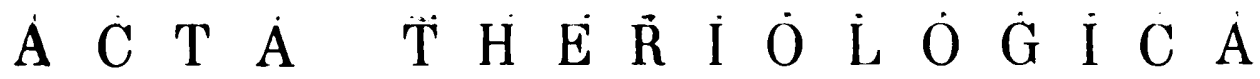

\begin{tabular}{lll} 
VOL. XI, 22: 449-484. BIAEOWIEŻA & $30 . X I I .1966$ \\
\hline
\end{tabular}

\author{
Zygmunt P I E L O W S K I*
}

\section{Forschungen über den Feldhasen. XIl. Die Raumstruktur der Population}

[Mit 6 Tabcllen und 11 Abb.]

Die Arbeit hatte zum Ziel, einige Elemente der Raumstruktur einer Hasenpopulation kennenzulernen, die ein stark urbanisiertes Vorstadtsgelände besiedelt, dass für eine gute Entwicklung des Hasenbestandes wenig geeignet ist. Im Ergebnis der Untersuchungen konnte festgestellt werden, dass im Areal der Population reguläre saisonale Migrationen der Hasen zwischen Wald und Feld stattfinden, die einen grossen Teil der Individuen betreffen. Forschungen über die Verteilung der Hasen in Abhängigkeit von den verschiedenen Feldkulturen ergaben, dass eine deutliche Bevorzugung deckunggebender Feldkulturen stattfindet. Die Untersuchungen machten auch eine Präferenz der Hasen bezüglich der verschiedenen Varianten des Feldmilieus ersichtlich. Weiter konnte eine detaillierte Differenzierung des Geländes in Abschnitte von verschieden grosser Tauglichkeit für den Hasen festgestellt werden. Schliesslich wurde ermittelt, dass bei der Verteilung der einzelnen Hasen im Gelärde Gruppierungstendenz besteht. Die festgestellten Anzeichen einer organisierten Raumstruktur der Hasenpopulation scheinen u.a. ein Resultat des fortdauernden Strebens danach zu sein, die einzelnen Individuen in möglichst geringen Masse Beunruhigungen und Gefahren auszusetzen, sind Ausdruck einer Adaptation der Population an die aktualle ökologische Situation im Lebensraum.

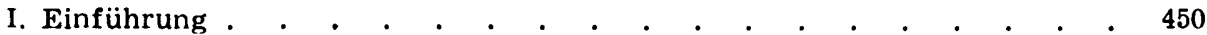

II. Untersuchungsgebict.$\quad$. . . . . . . . . . . . . . . . . 451

III. Methodik und Material . . . . . . . . . . . . . . . 452

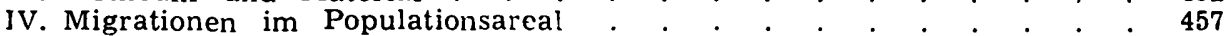

V. Räumliche Verteilung der Hasen und die Fcldkulturen . . . . . $\quad 463$

VI. Räumliche Verteilung đer Hasen und die Varianten des Feldmilieus . 465

VII. Ortswahl . . . . . . . . . . . . . . . . . . . . . . 468

VIII. Gruppierungstendenz.$\quad$. . . . . . . . . . . . . . 4473

IX. Diskussion der Ergebnisse . . . . . . . . . . . . . 478

X. Schlussfolgerungen für die Praxis . . . . . . . . . . . . . . 480 Schrifttum . . . . . . . . . . . . . . . . . . . 482

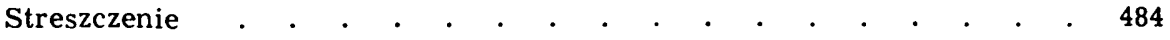

\footnotetext{
* Present address: Polish Hunting Ass., Research Station, Czempiń n/Poznań.
} 


\section{EINFÜHRUNG}

Die bisherigen das Problem der Gestaltung des Zahlenbestands der Population betreffenden ökologischen Forschungen uber den Feldhasen berücksichtigten beinahe garnicht die Frage der räumlichen Verteilung der Population. Dabei liegt es doch sehr nahe, dass die Raumstruktur, also die Art und Weise der Verteilung physiologisch und psychisch differenzierter Individuen im Areal der Population und die Form der räumlichen Ausnutzung dieses Areals durch die Individuen def Population - bei einer solchen Tierart wie der Hase, in wesentlichem Masse Einfluss auf das Los der Individuein und somit auf die Quantitätsdynamik der Population haben kann. Im ökologischen Schrifttum sind übrigens, abgesehen vor recht zahlreichen Arbeiten uiber dieses Problem, auch eine Reihe zusammenfassender Abhandlungen $\mathrm{zu}$ diesem Thema zu finden (z.B. Alle e et al., 1959; Naumov, 1963; Od u m, 1963; P etrus ew ic z, 1966).

In Abhängigkeit von den jokologischen Verhältnissen (Milieu und Biozönose) kain dic Raumstruktur der Population auf verschiedene Weise in Erscheinung treten und in verschiedenem Masse auf das Los der Individuen und der ganzen Population Einfluss haben. Petrusewicz (1966) weist beispielsweise auf eine ganze Reihe von Wegen hin, auf denen sich diese gegenseitigen Abhängigkeiten realisieren können. Einer von ihnen ist der modifizierende Einfluss der Struktur auf die Auswirkung der ökologischen Faktoren. In Abhängigkeit vom durch das Individuum eingenommenen Platz (besseren oder schlechteren), kann sich z.B. der Einfluss klimatischer Faktoren verschiedenartig auf sie auswirken. Auch können dic einzelnen Individuen verschieden stark Gefahren von Seiten ihrer Feinde ausgesetzt sein. Völlig verschiedenartig kann sich auch Aktivität und Migrationsgrad der Population wie auch die Populationsdichte (Individuenzahl auf Flächeneinheit des Areals) gestalten. Die Raumstruktur gibt den Individuen und Individuengruppen vielerlei Lebensmöglichkeit€n, verschieden grosse Überlebungschancen.

Der vorstehend angeführten These nach ist die Raumstruktur von den ökologischen Verhältnissen abhängig, in denen die Population lebt. Was den Hasen anbetrifft, dessen eigentlicher Lebensraum die freie, offenen Feldflur ist, so könnte man in. Ubereinstimmung mit der im einschlägigen Schrifttum herrschenden Vorstellung ( H e g e n d or f, 1933; B o b a c k, 1957; M ül le r - U s ing, 1962; R i e c k, 1963) die in Polen vorkommenden Haupttypen des Feldmilieus in einem Gradient unterschiedlicher ökologischer Verhältnisse von verschiedener Tauglichkcit für den Feldhasen aufstellen. Sehr günstige Lebensbedingungen findet der Hase vor allem auf solchem Feldgelände, auf dem Grossackerwirtschaft überwiegt, wo grosse Flächen offener Felder vorkommen und wo die menschlichen Siedlungen geschlossene Ortschaften bilden. Von solchen optimal für den Hasen günstigen Verhältnissen geht es dann über verschiedene Übergangsformen zu landwirtschaftlich genutzten Flächen, auf denen vorwiegend Kleinackerwirtschaft geführt wird, wo dementsprechend keine grösseren Flächen offener Felder vorkommen und wo die Agrarkultur ein niedriges Niveau hat. $\mathrm{Zu}$ solchen Typen von Feldmilieu kann auch das Vorstadtsgelände vieler Städte gezählt werden, dass manchmal zehntausende von Hektaren einnimmt und auch das Gelände ringsum grosse Industrieanlage:. Insgesamt gibt es in Polen verhältnismässig viel solchen Geländes, wobei das Tempo des Städtèoaus und der Indutralisierung des Landes vermuten lassen, dass in nicht allzu weiter Zukunft solche Art von Feldmilieu immer häufiger werden wird. Da diese Gebiete meistens jagdlich genutzt werden, ist das Prcblem der Erhaltung des Wildes in einer Siedlungsdichte, die überhaupt eine jagdlich zulässt, von grosser Bedeutung. 
Die vorliegende Arbeit hatte zum Ziel, einige Elemente der Raumstruktur einer Hasenpopulation kennenzulernen und zu ergründen, wie sich die Raumstruktur unter dem Einfluss ungünstiger ökologischer Verhältnisse gestaltet, die stark urbanisiertem Gelände eigen sind. Weiter ging es darum zu erforschen, ob Adaptationserscheinungen der Population an solche ökologische Situation bestehen und wie sich in $\mathrm{Zu}$ sammenhang damit der Zahlenbestand der Population formt.

Aus den Antworten auf diese Fragen erhoffte man gewisse praktische Hinweise hinsichtlich der in solchen Revieren zu führenden Jagdwirtschaft zu erhalten.

\section{UNTERSUCHUNGSGEBIET}

Die Untersuchungen wurden in einem zum Feldschutzgürtel des Kampinos-Nationalpark gehörenden Versuchsrevier durchgeführt. Das Revier liegt etwa $10 \mathrm{~km}$ nordwestlich von Warszawa, zwischen dem Ufer der Wisła und dem Rand des Kampinos-Waldes (Abb. 1). Das Gebiet umfasst ohne Berücksichtigung menschli-

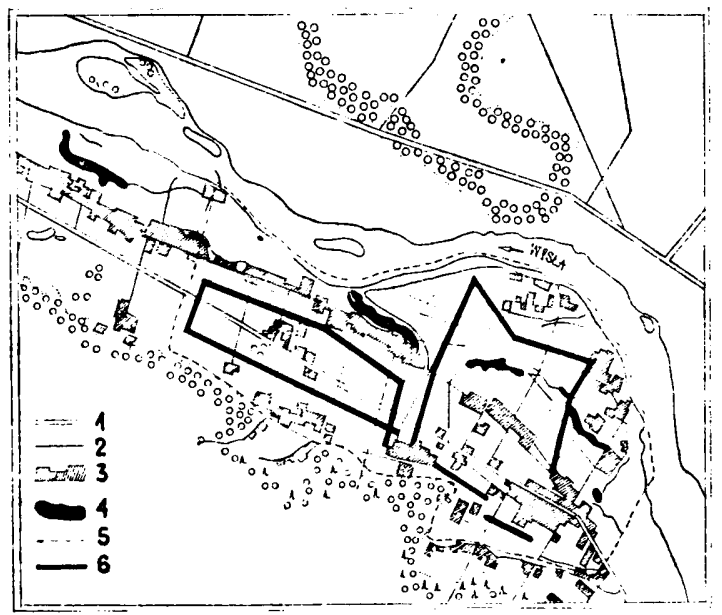

Abb. 1. Skizze des Untersuchungsgeländes. 1 - Fernverkehrstrasse, 2 - breitere Landwege, 3 - Ortschaften, 4 - Gewässer, 5 - Grenze des Untersuchungsgeländes, 6 - Taxationsstreifen.

cher Ansiedlungen, Autostrassen und Gewässer etwa 1900 ha. Auf einem Teil des Untersuchungsgeländes sind einige bis $\mathrm{zu} 100$ ha grosse Komplexe offener Felder anzutreffen. In der Nähe der Wisła kommt verhältnismässig viel Baumbestand in Form von reihenweise wachsenden Kopfweiden, Baum- und Strauchgruppen und einzelnen alten Pappeln vor. Stellenweise gibt es auch Obstgärten und in direkter Nähe des Stroms dichte Weidengebüsche und Pappelbestände. Auf diesem Teil des Untersuchungsgebiets befinden sich einige kleine Seen. Das ganze Gelände ist dicht bebaut. Ausser zwölf verschieden grossen Dörfern kommen zahlreiche vereinzelte Gehöfte und Häuser vor. 
Was das Grundeigentum anbetrifft, so kommt hauptsächlich Privatbesitz mit folgender Eigentumsstruktur vor: Bis zu 2 ha $-34 \%, 2-5$ ha $-37 \%, 5-10$ ha $24 \%$, $10-20$ ha $-5 \%$. Felder eines Staatsguts nehmen nur etwa 60 ha ein. Uberwiegend kommen leichte Böden vor. Die Bodennutzung gestaltet sich wie folgt: Ackerland - 48\%, Obstgärten - 1\%, Wiesen und Viehweiden - 14\%, Brachland $6 \%$, anderes Land $-31 \%$.

Durch die Mitte des Gebiets verläuft eine Autostrasse mit starkem Verkehr. Ausserdem ist das ganze Gelände von einem Landstrassennetz durchzogen, das ebenfalls von Fahrzeugen aller Art und von Fussgängern benutzt wird. Infolge der Eigentumsstruktur des Bodens - überwiegend Kleinbauernwirtschaft - ist der Menschenbetrieb auf den Feldern beinahe das ganze Jahr über sehr gross. Unabhängig davon ist hier zur Sommerzeit auch noch ein intensiver Ausflugsverkehr aus der nahen Grosstadt zu beobachten (geringe Entfernung zur Wisła, zum Wald und zu den Seen).

Das Revier wird stark von Jagdschädlingen aufgesucht. Eine im März 1965 durchgeführte Zählung der Hunde in den einzelnen Gehöften auf dem Untersuchungsgebiet gab im Ergebnis 983 notierte Hunde, wobei die tatsächliche Zahl zweifelsohne noch entschieden höher liegt und auf etwa 1100 Stück zu schätzen ist. Auch die Zahl streunender Katzen ist sehr hoch (Zählung der Katzen ist aus technischen Gründen schwer möglich). Die Hunde- und Katzenplage wird durch die grosse Zahl kleiner Gehöfte begünstigt. Es fehlt auch nicht an Nebelkrähen (Corvus cornix Lin na e us, 1758) und Elstern (Pica pica L in n e us, 1758). Es reicht zu erwähnen, dass allein im Jahre 1964 im Untersuchungsgelände 40 Krähennester entdeckt wurden. Die Nähe der Grosstadt und der Wisła hat auf diesem Gelände ausserdem noch eine enorme Penetration der Rabenvögel ausserhalb der Brutzeit zur Folge (Dobrowolski, et all, 1958; P inowski, 1959; Pinowski \& Wasilewski, 1962).

Das Gebiet ist Jagdrevier mehrerer Habichtspaare (Accipiter gentilis Linn a e us, 1758), die den nahen Kampinos-Wald bewohnen. Es konnte zwar fest gestellt werden, dass sich die Habichte hier vorwiegend von Tauben ernähren, aber ein gewisser Anteil ihrer Nahrung wird auch von jagdbaren Tieren gebildet (P i elowski, 1961). Von Greifvögeln, die als eventuelle Feinde des Niederwildes in Frage kommen, ist hier ausser dem Habicht noch der Mäusebussard (Buteo buteo

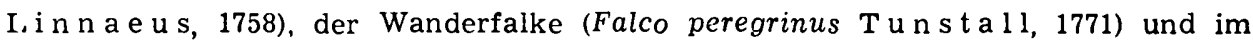
Winter auch der Rauhfussbussard (Buteo lagopus B r ünn ich, 1764) anzutreffen. Recht zahlreich kommt der Fuchs (Vulpes vulpes L in n a us, 1758) vor, der im nahen Wald und in den Weidengebüschen am Strom beheimatet ist. Auch Iltis (Mustela putorius Linnaeus, 1758) und Hermelin (Mustela erminea L in nae us, 1758) sind zu beobachten. Besonders dort wo Kopfweiden vorkommen, in denen sie ihre Schlupfwinkel haben.

\section{METHODIK UND MATERIAL}

Grundlägige Untersuchungsmethode war die Streifentaxation (J e z i erski \& Pielowski, 1958; Pielowski, 1962). Eine von sieben Leuten gebildete Treiberkette marschiert mit einem Marschtempo von ungefähr $4 \mathrm{~km}$ pro Stunde längs eines im Gelände abgesteckten Streifens von $100 \mathrm{~m}$ Breite und macht alle Hasen hoch, die auf diesem Streifen in der Sasse liegen. Auf entsprechenden Formularen werden dann alle Hasen notiert, wobei die Feldkultur angegeben wird, in der sie lagen. Notiert werden auch die ausserhalb des Taxationsstreifens hochwer- 
denden Hasen. Zum quantitativen Ergebnis der Taxation werden sie nicht gerechnet und stellen lediglich zusätzliches Material für andere Analysen dar.

Im Falle der vorliegenden Untersuchungen wurde als methodische Neuheit das Einzeichnen eines jeden hochgemachten Hasen auf einer Kartenskizze des Gcländes eingeführt. Auf diese Weise konnte jedesmal neben dem quantitativen Ergebnis auch das Verbreitungsbild der Hasen auf dem Taxationsstreifen erfasst werden. Der Taxationsstreifen war im Revier so abgesteckt, dass er ansich durch alle im Gelände vorkommeneden Varianten des Feldmilieus verlief (siehe Abb. 1). Steine Gesamtlänge betrug $19 \mathrm{~km}$, was umgerechnet 190 ha taxierter Fläche ausmacht, also $10 \%$ des gesamten Untersuchungsgebiet, und somit als relativ gut repräsentative Grösse für das ganze Revier angesehen werden kann.

S z e d e r je i (1958, 1959), der eine etwas andere Methode der Bestandsaufnahme für Hasenreviere empfiehlt, postuliert ebenfalls 1/10 der Gesamtfläche des gegebenen Reviers als repräsentative Probe. Auch Ruskov \& Petrov (1957), die Untersuchungen über die Brauchbarkeit verschiedener quantitativer Methoden für Hasenpopulationen durchgeführt haben, wandten Versuchsflächen an, die $10-15 \%$ der Gesamtfläche des Untersuchungsgebiets entsprechen.

Da der Taxationsstreifen durch die verschiedensten Varianten des Feldmilieus verlief, bestehen keine Ursachen zur Annahme, dass die Verhältnisse auf dem nicht taxierten Gelände wesentlich anders sein könnten. Ausserdem werden eventuelle Fehler durch das vielfache Wiederholen der Taxation weitgehend ausgeglichen.

Die Taxationen wurden in der Jahreszeit, in welcher sie überhaupt durchführbar sind, also in Durchschnittsjahren von Mitte September bis Mitte April, ansich einmal pro zwei Wochen ausgeführt. Dadurch wurde das Erfassen aller saisonalen Veränderungen in der Population gesichert und die Zufälligkeit der Ergebnisse verringert. Noch häufigere Taxationen erschienen nicht zweckmässig, da dann zu befürchten wäre, dass im Raumgefüge der Hasen auf dem taxierten Geländestreifen Störungen eintreten können. Die Taxationen wurden möglichst nur bei günstigen Wetterverhältnissen durchgeführt, d. h. bei Wetter ohne Niederschläge, starken Wind und bei Temperaturen nicht unter $-10^{\circ} \mathrm{C}$. In der Praxis war es nicht immer möglich, die zweiwöchentlichen Pausen einzuhalten. Es kamen nämlich Zeitspannen vor, wo die Wetterverhältnisse längere Zeit hindurch die Taxation unmöglich machten.

Angaben über die Zahl der durchgeführten Taxationen und die Zahl der notierten Individuen enthält Tabelle 1. In den Jahren 1961/621964/65 unterlag der Zahlenbestand der Hasenpopulation im Untersuchungsrevier einer deutlichen Verminderung. Die maximale, in diesem Crelände im Vorfrühling (März-April) beobachtete Siedlungsdichte wird von Jahr zu Jahr geringer:

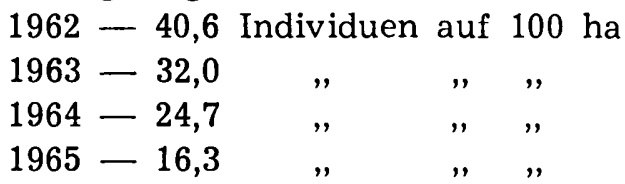

Die Bestandsverringerung von Jahr zu Jahr ist sehr regulär und beträgt jährlich ca $20 \%$ der Ausgangszahl aus dem Jahre 1962. In den Jahren 1962-65 verringerte sich die Maximaldichte also um etwa $60 \%$.

Was die Quantitätsdynamik in den einzelnen Jahren anbetrifft, so weist sie in der ersten Hälfte der Saison 1961/62, 1962/63 und 1963/64 
Tabelle 1.

Grösse des Materials aus den Streifentaxationen.

\begin{tabular}{|c|c|c|c|}
\hline \multirow[b]{2}{*}{5.180.} & \multirow{2}{*}{$\begin{array}{c}\text { Anzebl der } \\
\text { durcbgefühten } \\
\text { Taxationen }\end{array}$} & \multicolumn{2}{|c|}{ Anzabl der beobachteten Individuen } \\
\hline & & In Taxationastreisen & $\begin{array}{l}\text { Ausserhalb des } \\
\text { Toxationsstrelfens }\end{array}$ \\
\hline $1964 / I I-I V /$ & 5 & 235 & 21 \\
\hline $1961 / 62$ & 10 & 434 & 70 \\
\hline $1962 / 63$ & 12 & 448 & 58 \\
\hline $1963 / 64$ & 11 & 352 & 44 \\
\hline $1964 / 65$ & 8 & 155 & 33 \\
\hline Insgesamt & 46 & 1644 & 226 \\
\hline
\end{tabular}

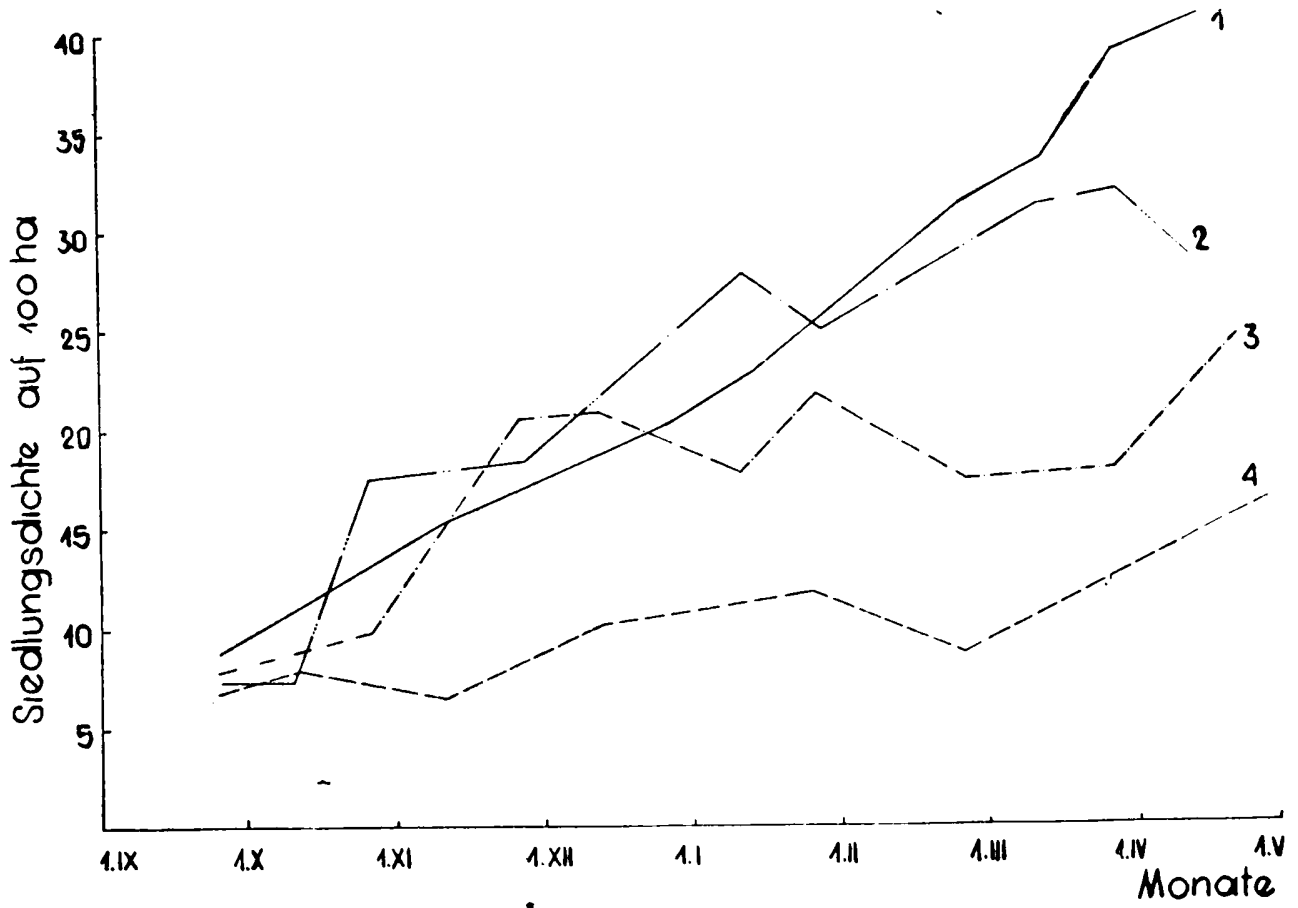

Abb. 2. Populationsdichte der Hasen - umgerechnet auf 100 ha Fläche des Taxationsstreifens unter Anwedung des „,beweglichen Mittelwertes” mit einem Sprung von zwei Wochen. $1-1961 / 62,2-1962 / 63,3-1963 / 64,4-1964 / 65$. 
grosse Ähnlichkeit auf. Das Quantitätsniveau liegt zur gleichen Zeit in der Saison 1964/65 schon deutlich tiefer (Abb. 2). Bedeutende Unterschiede der Siedlungdichte sind in der zweiten Hälfte der Untersuchungsaison der einzelnen Jahre sichtbar. Es muss hier jedoch unterstrichen werden, dass der Verlauf der Quantitätskurven absolut ein anderer ist, es normalerweise bei Funktionen solcher Art für Populationen von Tieren mit normalem Jahreszyklus der Fortpflanzung, zu denen wir auch den Feldhasen zählen, der Fall ist. Voll begründet ist die Vorstellung, dass die Jahreskurve in der Zeit vom Herbst bis zum Frühjahr ein Gefälle aufweisen muss, das bei fehlendem Zuwachs mit der Reduktion zusammenhängt (R i e ck, 1956; P e tr ov \& D ra g o e v 1962; S c a r la t e sc u, et al., 1963; R a c z yńs k i, 1964). Während des Sommers, also zur Vermehrungszeit der Hasen, sollte eine Bestandszunahme bis zu einem mehr oder minder dem Herbstbestand des Vorjahrs ähnelndem Niveau stattfinden, die dann anschliessend in eine Bestandsabnahme bis zu Frühjahrsminimum übergeht.

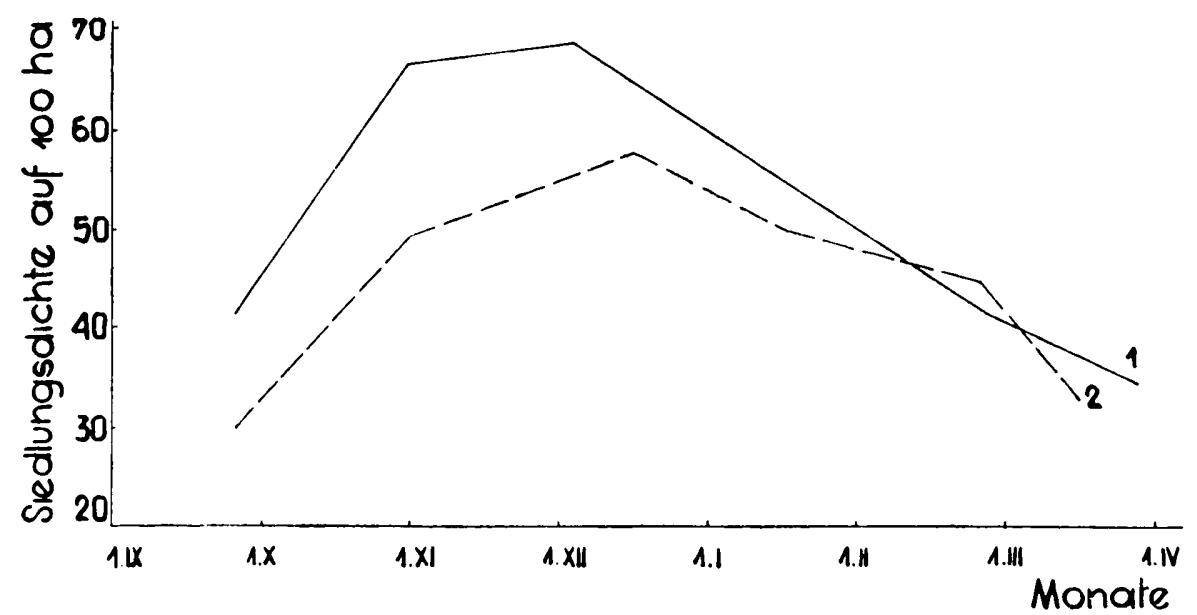

Abb, 3. Populationsaichte der Hasen im Hegerevier Moszna, Woiwodschaft Opole, umgerechnet auf 100 ha Fläche der Taxationsstreifens. $1-1957 / 58,2-1958 / 59$.

Es besteht die begründete Befürchtung, dass die Streifentaxation nicht in allen Jahrenzeiten ein glaubwürdiges Zahlenergebnis gibt. Die Untersuchungen von Jezie rski (in litt.) in der Woiwodschaft Poznań und eigene Forschungen in den Jahren 1958 und 1959 in der Woiwodschaft Opole haben erwiesen, dass die Ergebnisse der Streifentaxation im Herbst unter dem wirklichen Wert liegen. Das Quantitätsmaximum fällt in die Zeit gegen Herbstende und Winteranfang. Anstatt der schräge abfallenden Kurve erhält man also eine parabolische Kurve (Abb. 3) Noch weniger wahrscheinlich sehen die Quantitätskurven der untersuchten Hasenpopulation aus. Sie haben schräge ansteigende Tendenz (Abb. 2). 
Durch Analogic mit den oben erwähnten Untersuchungen in der Woiwodschaft Opole und den Untersuchungen von J e zie rski $(l$. c.) wird die Annahme begründet, dass die ständige herbstliche Zahlenzunahme der Hasen ein Artefakt ist und darauf beruht, dass zu einer Jahreszeit, wo auf den Feldern noch gute Deckung gebende Pflanzen wachsen, eben nicht alle Hasen von den Taxatoren hochgemacht werden. Im gleichen Masse, wie die Feldfrüchte von den Feldern verschwinden, nähert sich das Taxationsergebnis dem wirklichen Stand. Bis zum Anfang der nächsten Vegetationsperiode, also für Zentralpolen etwas bis Mitte April, muss die Streifentaxation die wirkliche Dichte der Hasen auf dem Taxationsstreifen erfassen. Es ist nämlich völlig unwahrscheinlich, dass die aus lärmenden Leuten bestehende Treiberkette, bei einem Abstand von $15 \mathrm{~m}$ von Mann zu Mann, auf der leeren Feldflur nicht sämtliche Hasen hochmacht, die sie auf ihrer Marschroute antrifft. Als Eestätigung dieser Vermutung können Angaben über die Entfernung des

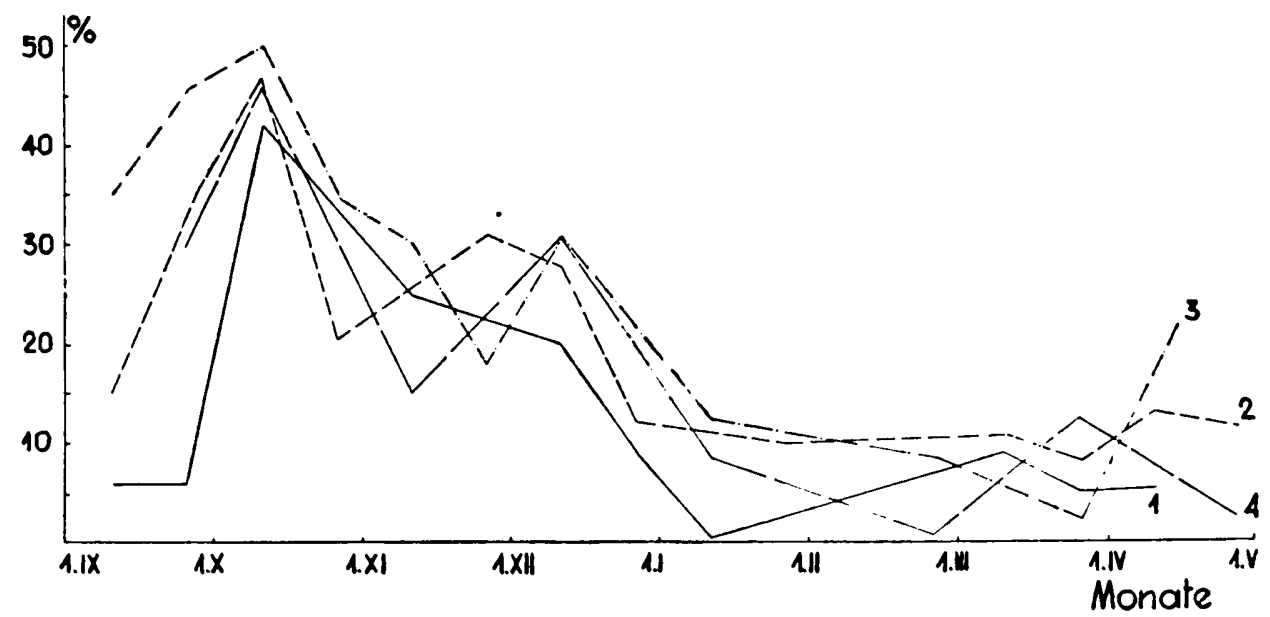

Abb. 4. Anzahl (in $\% \%$ ) der während der Streifentaxation in geringerer Entfernung als $5 \mathrm{~m}$ vor den Treiber hochwerdenden Hasen. 1 - 1961/62, $2-1962 / 63,3-1963 / 64$, $4-1964 / 65$.

Hochwerdens der Hasen vor der Treiberkette gelten. Während der Taxationen wurden die Entfernungen notiert, in welchen die einzelnen Hasen vor den Treibern hochwerden. Dabei erwies sich, dass im Herbst 50\% aller gezählten Hasen näher als $5 \mathrm{~m}$ vor der Treiberlinie hochwird. Später verringert sich dann dieser Prozentsatz allmählich, um sich ab Dezember auf einem Niveau von ungefähr $10 \%$ zu stabilisieren, dass dann bis zu Frühjahr unverändert bleibt (Abb. 4). Bei einer so geringen Fluchtdistanz im Herbst ist die Möglichkeit, dass ein gewisser Teil aller Individuen von den Treibern überhaupt nicht hochgemacht wird, sehr wahrscheinlich. 


\section{MIGRATIONEN IM POPULATIONSAREAL}

Im Untersuchungsgebiet ist in der Zeit vom Spätherbst bis zum Frühjahr eine fortwährende Zunahme der Siedlungsdichte der Hasen festzustellen (siehe Abb. 2), wo doch bei fehlendem natürlichen Zuwachs in der Population eine Quantitätsabnahme zu erwarten ist, die durch die natürliche Reduktion verursacht wird. Als einzige Erklärung dieser Erscheinung ist eine Einwanderung von Hasen aus anderen Gebieten annehmbar. Im Falle da die ganze Population einen relativ einheitlichen Lebensraum bewohnt, z.B. grosse Flächen offener Felder, schliesst sich eine solche Möglichkeit von selbst aus, da sogar dann, wenn man grössere Migrationen innerhalb der Hasenpopulationen, von denen man sagt, dass sie durch grosse Ansässigkeit charakterisiert sind (K o k e š, 1948; K oen e n, 1956; S z ed e r jei, 1958; B o b a ck, 1957; R i e ck, 1963), als möglich ansieht, bestehen keine Gründe zur Annahme, dass die Imigration grösser als die Emigration sein könnte.

Das Gelände in dem die vorliegenden Untersuchungen durchgeführt wurden hat jedoch solchen Charakter, dass durch Migration verursachte Quantitätsveränderungen im Jahreszyklus möglich sind. Seine topographische Gestaltung bewirkt, dass es in grossem Masse von anderen grösseren Feldfluren isoliert ist (siehe Abb. 3). Von nördlicher Seite besitzt es eine natürliche, von der Wisła gebildeten Grenze. Von Osten ist das Vorstadtgelände von Gross-Warszawa Grenze und von Süden der Rand eines grossen Waldkomplexes - der Puszcza Kampinoska. Lediglich die westliche Grenze verläuft auf einem verhältnismässig kurzem Abschnitt durch offene Felder. Die dort ausserhalb des eigentlichen Untersuchungsgebiets des Forschungsreviers verbleibenden ca 1.500 ha Felder grenzen ebenfalls mit der Wisła, dem Kampinos und mit ausgedehnten, feuchten Wiesen. Das dortige Gelände unterscheidet sich also in keiner Weise vom Untersuchungsgebiet. Die Ergebnisse einiger dort durchgeführten Streifentaxationen bestätigen diese Vermutung. In der Saison 1961/62 und 1963/64 wurden dort je 3 Taxationen von $10 \mathrm{~km}$ Länge gemacht (im Herbst, im Winter und im Frühjahr). Die registrierte Siedlungsdichte auf 100 ha weicht nur ganz gering von der zur gleichen Zeit im Untersuchungsgebiet festgestellten Bestandsdichte ab.

Was die Isolation des Untersuchungsgebiets anbetrifft, so ist der grosse Strom für die Hasen ansich ein nicht zu bewältigendes Hindernis. Zwar sind Fälle bekannt, dass Hasen breitere Gewässer durchschwommen. Berücksichtigt man jedoch, dass die Wisła unterhalb von Warszawa recht starke Strömung hat und durchschnittlich $300 \mathrm{~m}$ breit ist, dann wird die Wahrscheinlichkeit regulärer Durchquerung dieses Gewässers durch eine grössere Anzahl von Hasen doch sehr gering. Im Winter kommen zwar Zeitabschnitte vor, in den der ganze Strom vereist ist und dann ist der 
Ubbergang von Ufer zu Ufer natürlich möglich. Gewöhnlich hält sich die Eisdecke jedoch nicht länger als 4-6 Wochen und es kommen Jahre vor, in denen der Strom überhaupt nicht zufriert. Abgesehen davon ist das Gelände jenseits des Flusses sehr dem diesseitigen ähnlich, da es auch zur Zone des Urbanisierungseinflusses Warszawas gehört. Vermutlich ist es also für den Hasen ebenfalls wenig als Lebensraum geeignet.

Auch das Stadtgebiet von Warszawa stellt als Grenze ein absolutes Hindernis dar. Geht man aus der Vermutung heraus, dass die Zunahme der Siedlungsdichte in der Zeit vom Spätherbst bis zum Frühjahr durch Einwanderung entsteht, was übrigens die einzige sinnvolle Erklärung dieser Erscheinung wäre, dann bleibt nur die Möglichkeit grosser Migrationsbewegungen zwischen dem Wald und den Feldern. Die Waldgrenze macht $1 / 3$ der Gesamtgrenze des Forschungsreviers aus. Das Waldgebiet von etwa 20.000 ha Grösse weist eine grosse Biotopsmosaik auf, kann aber insgesamt betrachtet, nicht als optimaler Lebensraum des Feldhasen angesehen werden. Aufgrund eines allgemeinen, auf vieljährige Zufallsbeobachtungen und auf Angaben aus anderen im Wald durchgeführten Untersuchungen basierenden Eindrucks, kann die Siedlungsdichte hier als höchstens mittelmässig bezeichnet werden. Konkretes, in der Zeit vergleichbares Zahlenmaterial darüber ist jedoch nicht vorhanden. Die Methoden der Bestandsaufnahme von Hasen in Waldgebieten sind aussergewöhlich zeitraubend und verlangen den Einsatz einer grossen Treiber- und Beobachterzahl, beziehungsweise sind nur im Winter bei Neuschnee durchführbar (Rusk ov \& Petrov, 1957).

In den Jahren 1963, 1964 und 1965 wurden im Wald auf mehreren in F'eldnähe gelegenen Versuchsflächen von insgesamt 45 ha Grösse, Winterbestandsaufnahmen durch Zählung der Hasenspuren im Neuschnee durchgeführt, die folgende Ergebnisse gaben:

28. Januar $1963-123$ Hasen, umgerechnet auf 100 ha

4. Februar $1964-10 \mathrm{\jmath}$

30. Januar $1965-65$

Das ist eine sehr grosse Dichte, die ganz gewiss nicht das ganze Jahr über anhält. In der hauptsächlich aus Kieferndickungen bestehenden Randzone des Waldes muss es zu einer zeitweiligen Konzentration der Hasen gekommen sein. Bezeichnend ist jedoch die Abnahme der Dichte von Jahr zu Jahr, analogisch zur Quantitätsabnahme im Feldrevier. Methodische Schwierigkeiten machten jedoch ein laufendes Verfolgen der Bestandsschwankungen im Walde sogar auf den kleinen Versuchsflächen unmöglich. Die ganze Theorie über die saisonalen Wanderungen der Hasen zwischen Wald und Feld basiert also beinahe ausschliesslich auf Zahlenmaterial aus dem Feldrevier. Volle Bestätigung wäre erst durch parallele Angaben auch aus dem Walde möglich. 
Wie kann man sich die Dynamik dieser Migrationen vorstellen? Auf dem Feldgelände findet im Winter eine normale natürliche Reduktion des Bestandes zum Ausgangsstand vor der Vehrmehrungsperiode statt. Die regelrechte Quantitätskurve muss also zu dieser Zeit einen schräge abfallenden Verlauf haben (siehe Abb. 3). Theoretische gesehen müsste sich auch im Wald die Situation ähnlich gestalten. Im Spätwinter und Vorfrühling findet jedoch eine ständige Emigration des Hasen aus dem Wald auf die in der Nähe gelegen Felder statt. Die Emigration ist so stark, dass in dem isolierten, verhältnismässig kleinen Feldrevier, trotz der natürlichen Reduktion des Bestands, dessen Dichte ständig ansteigt. Gleichzeitig vermindert sie sich natürlich mit verstärktem Tempo im Walde. Im Sommer und Herbst haben wir es dann mit einer umgekehrten Erscheinung zu tun.

Aufgrund des zur Verfügung stehenden Materials kann ein Versuch gemacht werden, die vorstehend beschriebene Erscheinung saisonaler Wanderungen in quantitative Rahmen zu erfassen. Aus der Quantitätskurve der Population in den einzelnen Jahren kann man die theoretische Keduktionskurve des Bestandes im Winter und Vorfrühling ableiten. Dem Verlauf der Quantitätskurven von Populationen aus anderen typischen Feldrevieren nach zu urteilen (eigene Untersuchungen und J ezi e r ski, in litt.), könnte man annehmen, dass diese Reduktion etwa $50 \%$ des spätherbstlichen Stands ausmacht, wobei allerdings kleinere oder grössere Abweichungen in Kauf genommen werden müssen. Diese Angaben können insofern nicht ohne weiteres auch das Untersuchungsrevier bezogen werden, da sie Populationen von grosser Bestandsdichte betreffen, die jagdlich nicht durch Abschuss, sondern durch Fang lebender Hasen bewirschaftet werden. Ausserdem ist die Grösse der Reduktion nicht in jedem Jahr gleich gross, da sie von vielen veränderlichen Faktoren abhängt. Darauf weisen die wenn auch nur zweijährigen Angaben aus der Woiwodschaft Opole hin (siehe Abb. 3), wo die Reduktion in Jahre 1958 beinahe $50 \%$ des spätherbstlichen Bestands ausmacht, im Jahre 1959 dagegen nur $42 \%$. An drze jew ski \& Jezierski (1966), die die Grösse der natürlichen Reduktion einer von ihnen untersuchten Hasenpopulation auf völlig andere Weise bestimmt haben, bezeichnen sie auch mit $40-50 \%$ des Bestands. Um die Möglichkeit zu vermeiden, einen groben Fehler zu begehen, könnte man für die untersuchte Population bei der Bestimmung der Reduktion eine grosse Spannweite, z.B. von 25 bis $50 \%$ anwenden. Da jedoch die Zahl, die die Grösse der Reduktion bezeichnet, im Vergleich zur Zahl, welche die Migrationsintensität zwischen Wald und Feld charakterisiert, verhältnismässig gering ist, erscheint das Überprüfen mehrerer alternativer Versionen unnotwendig. Die Annahme einer grösseren oder kleineren Reduktion ist in 
dieser Situation für die Grósse der Zahl, deren Bestimmung die vorliegenden Erwägungen gelten, unwesentlich. Der Einfachheit halber wurde die durchschnittliche Grösse der Reduktion mit 30\% des spätherbstlichen Zahlenbestands der Population festgelegt.

Der Unterschied zwischen dem tatsächlichen Stand und der theoretischen Reduktionskurve, plus die für zweiwöchentliche Zeitabschnitte berechnete Reduktionsgrösse für die Migranten (es muss vorausgesetzt werden, dass auch sie der Reduktion unterliegen und das wahrscheinlich mindestens im gleichen Masse wie der ansässige Teil der Population), bezeichnet die Migrationsintensität (Abb. 5). Die Angaben betreffen die

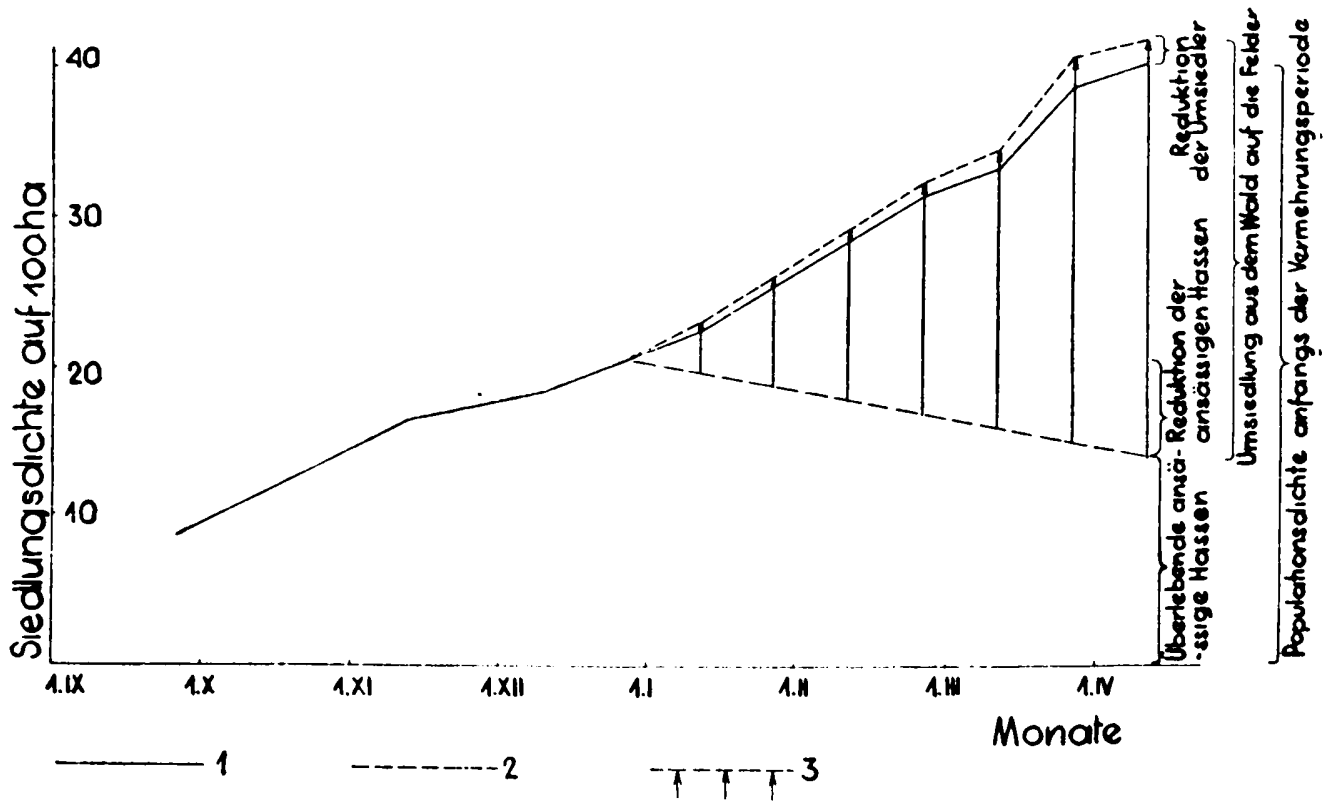

Abb. 5. Migrationsintensität der Hasen zwischen Wald und Feld im Winter und Frühjahr 1962. 1 - Populationsdichte, 2 - natürliche Reduktion, 3 - Migrationsintensität.

Zeit von Mitte Dezember bis April, also einen Zeitabschnitt, zu welchem die fortwährende Zunahme der beobachteten Anzahl an Hasen schon nicht mehr durch methodische Momente zu erklären ist (siehe Methodik). Bei den Berechnungen wurde folgende Formel angewandt:

$$
\mathrm{N}=(\mathrm{A}-\mathrm{a})\left(1 \frac{\mathrm{b}}{\mathrm{a}}\right)
$$

wo: N - Migrationsintensität, A - Siedlungsdichte der Hasenpopulation, a - Dichte des ansässigen Teils der Hasenpopulation, b - Grösse der Reduktion des ansässigen Teils der Population bezeichnen. 
Entsprechende Angaben für die Saison 1961/62 enthält Tabelle 2. Aus einer solchen Berechnung erhält man im Ergebnis, dass im Zeitabschnitt Winter-Frühjahr dieser Saison auf je 100 ha Feldrevierfläche ca 28 Hasen aus dem Wald dazukommen sind. Umgerechnet auf die Gesamtfläche des Untersuchungsgebiets gibt das etwa 540 Stück Hasen, die aus dem Wald auf die Felder umgesiedelt sind. Mindestens genau so viel Individuen und aller Wahrscheinlichkeit nach bedeutend mehr, haben natürlich im Sommer und Frühherbst eine umgekehrte Wanderung in den Wald machen müssen.

Für das Jahr 1963 geben analogische Berechnungen im Ergebnis ca 16 migrierender Hasen pro 100 ha, für das Jahr 1964 - 12 Hasen und für das Jahr 1965 - 10 Hasen. Die Zahl der Migranten wird von Jahr zu Jahr kleiner. Für eine Veränderung der Migrationsintensität spricht auch die Tatsache, dass die Quantitätskurven in der ersten Phase ihres Verlaufs in den einzelnen Jahren grosse Úbereinstimmung aufweisen (siehe Abb. 2). Es wirft sich hier der Verdacht auf, dass dieses Bild ein Resultat der angewandten Methodik sein kann, die - wie schon gesagt im Herbst nicht den tatsächlichen Zahlenbestand der Population erfasst. Es ist jedoch unwahrscheinlich, dass diese Tatsache Einfluss auf die Zahl der im Herbst jedes Jahres registrierten Hasen haben kann, da die Zahl der von den Treibern hochgemachten Individuen immer proportionell zur tatsächlichen Siedlungsdichte sein muss.

Die Siedlungsdichte ist also im Herbst und Winteranfang eines jeden Jahres ähnlich. Unterschiedlich wird sie erst in den Monaten von Januar bis April also dann, wenn die Hasenwanderungen aus dem Wald auf die Felder stattfinden. Der von Jahr zu Jahr kleinere Ausgangsstand vor der Vermehrungsperiode zieht in der Konsequenz einen geringeren natürlichen Zuwachs im gegebenen Jahr nach sich. Die Untersuchungen erwiesen jedoch, dass trotz bedeutender Unterschiede im Frühjahrs-Ausgangsniveau, die Siedlungsdichte in der zweiten Septemberhälfte jedes Jahres beinahe die gleiche ist. Das könnte davon zeugen, dass zu dieser Zeit der Lebensraum im Untersuchungsgebiet ein bestimmtes Fassungsvermögen besitzt, das wie er scheint, dadurch bedingt wird, dass die Hasen zu dieser Zeit besonders starker Beunruhigung und besonders vielen Gefahren ausgesetzt sind. In dieser Situation limitieren die Verhältnisse des Lebensraums unabhängig vom Verlauf der Vermehrungsperiode und von der Grösse der Zuwachsrate, auf den Feldern im Spätsommer und Herbst eine niedrige Siedlungsdichte der Population. Der gesamte Überschuss, der im Zusammenhang mit der allgemeinen Quantitätsverminderung der Population mit jedem Jahr kleiner wird, ist dazu gezwungen in den Wald abzuwandern. Im Winter, wenn auf den Feldern wieder Ruhe 
Tabelle 2.

Berechnung der Migrationsintensität der Hasen zwischen Wald und Feld (umgerechnet auf 100 ha Fläche) in der Saison 1961/62, bei einer 30\% betragenden natürlichen Reduktion des spätherbstlichen Bestandes.

\begin{tabular}{|c|c|c|c|c|c|c|c|}
\hline \multirow[t]{2}{*}{ zof taboobnit t } & \multirow[t]{2}{*}{$\begin{array}{l}\text { s1edlungs- } \\
\text { d1chte }\end{array}$} & \multirow{2}{*}{$\begin{array}{l}\text { Sledlungsdichte } \\
\text { des ansäss1gen } \\
\text { Te1ls }\end{array}$} & \multicolumn{2}{|c|}{$\begin{array}{c}\text { Grösse der Reduktion } \\
\text { des ansassigen } \\
\text { Tells }\end{array}$} & \multirow{2}{*}{$\begin{array}{c}\text { Effekt1ve } \\
\text { Zunabme der } \\
\text { Umsledler- } \\
\text { zabl }\end{array}$} & \multirow{2}{*}{$\begin{array}{c}\text { Grösse } \\
\text { der Reduction } \\
\text { der } \\
\text { Unsledler }\end{array}$} & \multirow{2}{*}{$\begin{array}{l}\text { Zunahme } \\
\text { der } \\
\text { M1grations- } \\
\text { Intensitäț }\end{array}$} \\
\hline & & & Anzahl & $\$$ & & & \\
\hline & 1 & 2 & 3 & 4 & 5 & 6 & 7 \\
\hline & & & & $3: 2$ & $1-2$ & $1 / 5 \times 4 /: 100$ & $5+6$ \\
\hline $16 / 31 \times I I$ & 20,2 & 20,2 & 0 & 0 & 0 & 0 & 0 \\
\hline $1 / 15 I$ & 22,7 & 19,4 & 0,8 & 4,1 & 3,3 & 0,1 & 3,4 \\
\hline $16 / 31 I$ & 25,3 & 18,5 & 0,9 & 4,9 & 7,0 & 0,3 & 7,3 \\
\hline $1 / 49$ II & 28,5 & 17,6 & 0,9 & 3,1 & 10,9 & 0,6 & 11,5 \\
\hline 16/28 II & 31,6 & 16,7 & 0,9 & 5,4 & 14,9 & 0,8 & 95,7 \\
\hline $1 / 19$ III & 33,4 & 15,8 & 0,9 & 5,7 & 17,6 & 1,0 & 48,6 \\
\hline $16 / 34 I I I$ & 39,3 & 14,9 & 0,9 & 6,0 & 24,4 & 1,3 & 25,9 \\
\hline $1 / 4317$ & 40,6 & 14,0 & 0,9 & 6,4 & 26,6 & 1,7 & 28,3 \\
\hline
\end{tabular}


herrscht, wird das Fassungsvermögen wieder grösser. Dann erfolgt die Zurückwanderung der Hasen aus dem Wald auf die Felder.

In der Saison 1964/65 fand ich ein bedeutsames Zusammenbrechen des Dichteniveaus der Population statt. Der Zahlenbestand des ansässigen Teils der Population ist niedrig von Anfang an und bei nur geringer Reemigration aus dem Wald, was übrigens aufgrund des niedrigen Ausgangsstandes im Frühjahr 1964 vorauszusehen war — findet auch die in den Vorjahren festgestellte Zunahme der Siedlungsdichte im Winter-Frühjahr Zeitabschnitt nicht statt.

Diese Angaben können natürlich nicht auf volle Genauigkeit präten- dieren, da die Berechnungen notgedrungen eine gewisse Zahl von Unbekannten oder rein theoretisch durch Vergleich mit der Situation in anderen Revieren erhaltene Vermutungen zulassen müssen. Wenn auch bei der Bestimmung dieser Unbekannten eine ziemlich grosse Willkür vorauszusetzen ist, so scheint die Tatsache saisonaler Migrationen eines Teils der Population doch keinem Zweifel zu unterliegen.

\section{RÄUMLICHE VERTEILUNG DER HASEN UND DIE FELDKULTUREN}

Das zu Verfügung stehende Beobachtungsmaterial aus den Streifentaxationen erlaubt es u.a. sich zu orientieren, ob bei den Hasen der untersuchten Population eine Auswahl in Bezug zu den einzelnen Arten von Feldkulturen besteht. Es wäre durchaus denkbar, dass eine Verbindung zwischen der räumlichen Verteilung der Population und der Feldmosaik besteht und dass die Verteilung zu verschiedenen Jahreszeiten, in Abhängigkeit von der phänologischen Situation eine andere sein wird. Eine solche Überzeugung ist übrigens sehr unter den Jägern verbreitet.

Es wurden drei grundsätzliche Kategorien von Feldkulturen unterschieden: Wintersaat, Pflugacker und andere Feldfrüchte. $\mathrm{Zu}$ der letzten Kategorie, die im Winter nur einen minimalen Prozentsatz der Gesamtfläche der Felder ausmacht, gehören ausser Futterpflanzen und Hackfrüchten auch Wiesen, Viehweiden, Obstgärten, Gebüsche und Gehölze wie auch krautbewachsenes Brachland. Die Analyse des diesbezüglichen Materials konnte zusammen für dreieinhalb Jahre Untersuchungszeit durchgeführt werden, da wie aus entsprechenden Tabellen und Funktionen hervorging, die Auswahl der Hasen bezüglich der einzelnen Feldkulturen in allen Jahren die gleiche war. Lediglich die Saisan 1964/65 wurde nicht mit einbezogen, da die zu geringe Quantität der Hasen in diesem Zeitabschnitt die Möglichkeit sehr grosser Zufälligkeit der Ergebnisse schaffte. Die Angaben betreffen insgesamt 1.682 Hasenbeobachtungen wobei auch Individuen berücksichtigt wurden, die ausserhalb des Taxationsstreifens hochgeworden sind. 
Auffallend ist die Stabilisierung des Verteilungsgefüges in den Monaten von Dezember bis April. Nach den jähen herbstlichen Veränderungen des Vorkommens der Hasen in den einzelnen Typen von Feldkulturen, die mit dem Verschwinden der Hackfrüchte und Grünfutterpflanzen und Erscheinen von Wintersaat und Pflugacker übereinstimmen, festigt sich ein Ausnutzungsniveau aller drei Kategorien von Feldkulturen, dass dann bei nur geringen Abweichungen unabhängig von verschiedenen anderen Faktoren, wie z.B. Wetterverhältnisse, im Januar anfangende Paarungszeit, in diesem Revier festgestellte Hasenwanderungen zwischen Wald und Feld - bis zum Frühjahr in etwa gleicher Höhe anhält (Abb. 6).

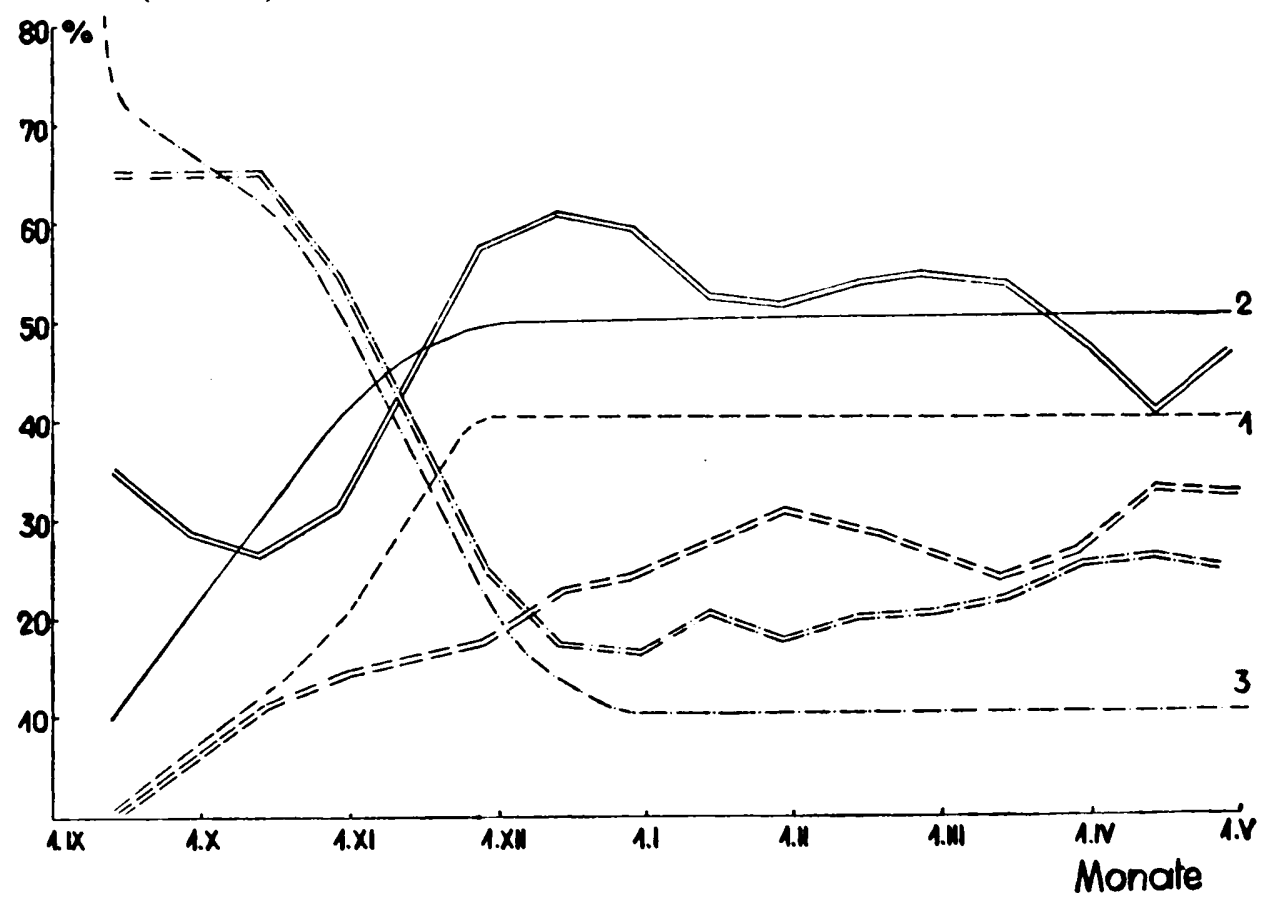

Abb. 6. Vorkommen der Hasen auf den einzelnen Feldkulturen (in $0 \% 0^{\prime} / 0$ ), Mittelwert für die Jahre 1961-1964. 1- Wintersaat, 2 - Pflugacker, 3 - andere Kulturen. Durch Doppellinie wird das Vorkommen in den einzelnen Feldkulturen, durch einfache Linie der tatsächliche prozentuale Anteil der verschiedenen Feldkulturen (nach Angaben der Abt. Landwirtschaft im Rat des Kreises) bezeichnet.

Wird dieses Problem unter Berücksichtigung des Anteils der einzelnen Kategorien von Feldkulturen näher erwogen (die entsprechenden Angaken erhielten wir von der Abteilung Landwirtschaft im Rat des Kreises Nowy Dwór Maz.) ist festzustellen, dass nur beim Pflugacker seinem tatsächlichen prozentualen Anteil auch der dort angetroffene Anteil der Gesamtzahl an Hasen entspricht. Bezüglich der Wintersaat weist ein 
Vergleich dieser beiden prozentualen Funktionen darauf hin, dass die Saaten von den Hasen während der ganzen Saison ziemlich gemieden werden. Die Anzahl der auf den Wintersaaten angetroffenen Hasen ist bedeutend kleiner, als sie sein müsste, wenn keine Auswahl stattfinden würde. Umgekehrt ist es mit den sogenannten ,,anderen" Feldfrüchten. Insgesamt nehmen sie flächenmässig etwa $10 \%$ des ganzen Reviers ein, wogegen der Prozent der dort angetroffenen Hasen entschieden höher liegt (Abb. 6).

Inwiefern also die Verbreitung der Hasen im phänologischen Aspekt in den ,anderen" Feldkulturen ein stabiles Gefüge aufweist, insofern tritt in Bezug zu den einzelnen Kategorien von Feldkulturen überhaupt, eine Auswahl zugunsten von deckungsgebenden Feldbiotopen auf. Das Suchen nach Deckung scheint hier der ausschlaggebende Moment dieser Auswahl zu sein. Ubrigens stammen die entsprechenden Angaben ja gerade von Beobachtungen solcher Hasen, die aus den Sassen hochwurden, in denen sie sich bei Tage aufhalten. Die Nahrungsbasis spielt in diesem Falle wohl keine Rolle. Es ist möglich, dass hier der klimatische Faktor entscheidende Bedeutung hat und mit Bestimmtheit auch die Frage der vor Feinden schützenden Deckung.

Das Problem der Platzwahl für die Sasse ist gewiss ein recht wichtiges Glied in der Gesamtheit des Gefüges der räumlichen Verteilung der Hasenpopulation.

\section{RÄUMLICHE VERTEILUNG DER HASEN UND DIE VARIANTEN DES FELDMILIEUS}

Man kann vermuten, dass die Eigenschaften des Feldmilieus auch einen nicht weniger wichtigen Faktoren für die Verteilung der einzelnen Individuen der Hasenpopulation darstellen. In Abhängigkeit von der topographischen Gestaltung des Geländes und von dessen Charakter, können in diesem Milieu eine ganze Reihe verschiedener Geländevarianten unterschieden werden, die für den Hasen bestimmt nicht ohne Bedeutung sind.

Bei den vorliegenden Untersuchungen wurden vier verschiedene Varianten des Feldmilieus unterschieden, von denen anzunehmen war, dass sie in diesem Revier einen wesentlichen Einfluss auf das Bild der räumlichen Verteilung der Hasenpopulation haben können. Das wären offene Felder, Felder in Waldnähe (Entfernung vom Wald nicht grösser als $500 \mathrm{~m}$ ), Felder in Dorfnähe und Felder mit Baumbestand (Obstgärten, Reihen von Kopfweiden, Pappelgehölze usw.).

Theoretische Grundlage einer Analyse der Verteilung der Hasen in Abhängigkeit von der Geländegestaltung war die Vermutung, dass in Abhängigkeit von der Jahreszeit, den phänologischen Verhältnissen, der 


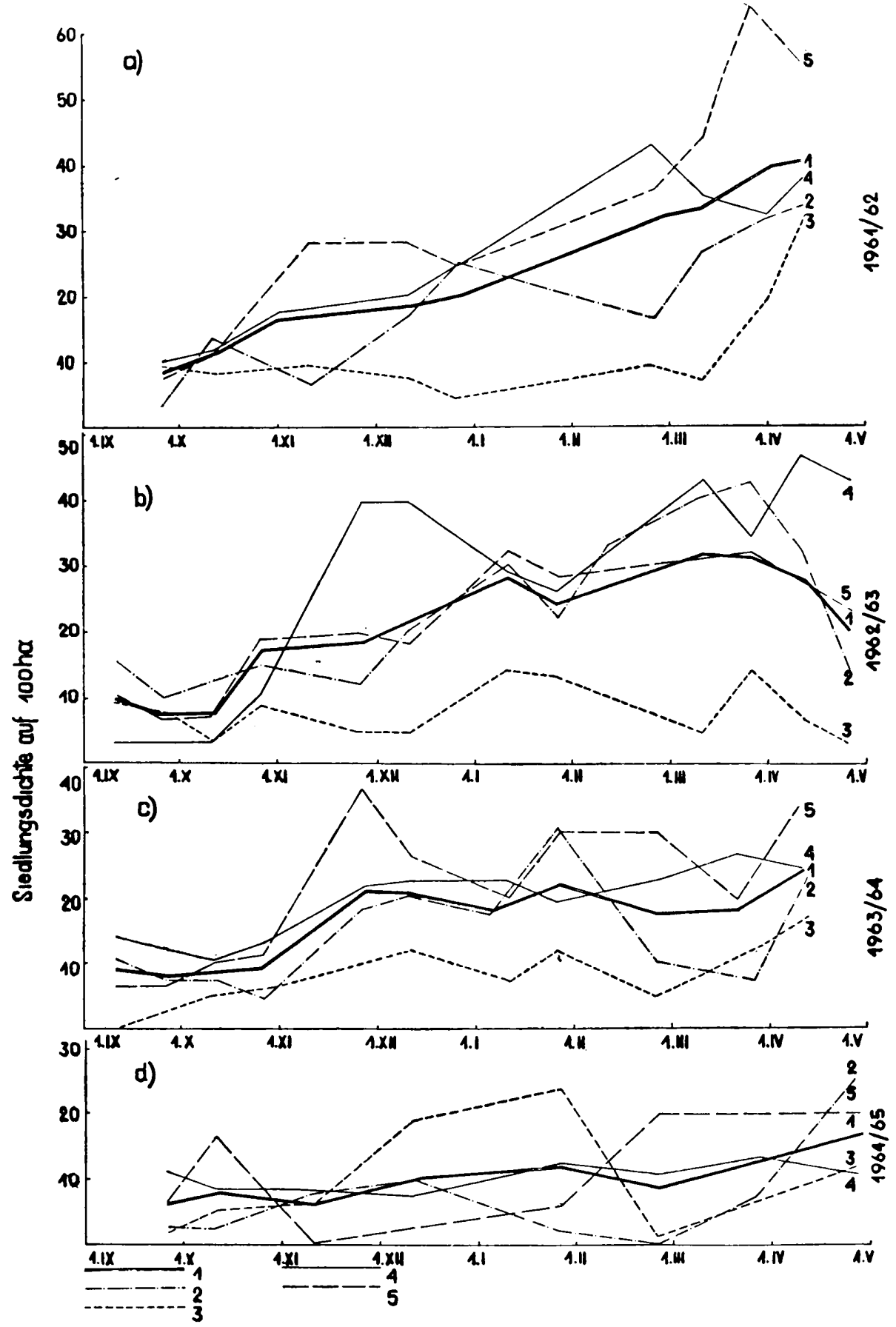

Abb. 7. Siedlungsdichte (auf 100 ha) in den einzelnen Varianten des Feldmilieus (bei Anwendung des ,beweglichen Mittelwertes” mit einem Sprung von zwei Wochen). 1 - gesamtes Untersuchungsgebiet, 2 - Felder in Waldnähe, 3 - Felder in Dorfnähe, 4 - offene Felder, 5 - Felder mit Baumbestand. 
I'opulationsquantität und einer Reihe anderer Faktoren, die Siedlungsdichte der Hasen in verschiedenen Typen des Feldmilieus unterschiedlich sein kann. Das zur Verfügung stehende Material aus den Streifentaxationen ermöglicht eine solche Analyse. Flächenmässig sind sich die einzelnen Milieuvarianten nicht gleich. Auch die durch sie verlaufenden Abschnitte der Taxationsroute haben nicht die gleiche Länge. Die Ergebnisse wurden also auf 100 ha Feldfläche umgerechnet, was einen clirekten Vergleich möglich macht. Das Material wurde getrennt für die einzelnen Untersuchungsjahre zusammengestellt (Abb. 7a, b, c, d; 'Tab. 3).

In den ersten drei analysierten Jahren ist die Siedlungsdichte der Hasen auf Feldern in Dorfnähe in den einzelnen Monaten deutlich niedriger als die durchschnittliche Populationsdichte für das ganze Revier. Das

Tabelle 3.

Abweichung der Siedlungsdichte der Hasen (auf 100 ha) in den einzelnen Varianten des Feldmilieus von der durchschnittlichen Populationsdichte für das gesamte Untersuchungsgebiet.

\begin{tabular}{|c|c|c|c|c|c|}
\hline \multirow[b]{2}{*}{$s$ a 1 s o n } & \multicolumn{4}{|c|}{ Varlanten des Feldmilieus } & \multirow{2}{*}{$\begin{array}{l}\text { Für das } \\
\text { gianze } \\
\text { Gebiet }\end{array}$} \\
\hline & $\begin{array}{l}\text { Felder in } \\
\text { Waldnähe }\end{array}$ & $\begin{array}{l}\text { Offene } \\
\text { Feldes }\end{array}$ & $\begin{array}{l}\text { Felder mit } \\
\text { Baum- } \\
\text { bestand }\end{array}$ & $\begin{array}{l}\text { Feluer in } \\
\text { Dorfnähe }\end{array}$ & \\
\hline $1961 / 52$ & 1,43 & 1,09 & 0,95 & 0,53 & 23,7 \\
\hline $1962 / 63$ & 1,10 & 1,11 & 1,37 & 0,43 & 19,5 \\
\hline $1963 / 64$ & 0,99 & 1,11 & 1,34 & 0,55 & 16,9 \\
\hline $1964 / 65$ & 0,74 & 1,07 & 1,18 & 1,00 & 10,0 \\
\hline
\end{tabular}

zcugt davon, dass die in der Nähe von Dörfern und Gehöften gelegenen Felder von den Hasen gemieden werden. In der Saison 1964/65 wiederholte sich diese Gesetzmässigkeit nicht. In den Monaten Dezember und Januar war die Dichte der Hasen auf den dorfnahen Feldern $\in$ ntschieden hoher, als auf allen anderen Feldern (siehe Abb. 7d). Das diese Erscheinung verbildliche Material ist jedoch sehr gering, so dass ihm kaum grössere Beachtung gewidmet werden kann.

Am nächsten der Gesamtdichte ist der Zahlenbestand der Hasen auf effenen Feldern. Die Kurven beider Werte haben in den einzelnen Jahren cinen sehr ähnlichen Verlauf. Die Durchschnittsdichte auf diesen Feldern zeigt von Jahr zu Jahr grosse Ständigkeit und hält sich in jedem Jahr 
auf einem Niveau von ca $10 \%$ der Durchschnittsdichte für das gesamte' Gebiet. Die Besiedlung dieses Feldtyps ist weitgehend ausgeglichen (Tabelle 3). Die Dichte auf Feldern mit Baumbestand und auf Feldern in Waldnähe unterliegt bedeutenden Schwankungen, die übrigens in den einzelnen Jahren weder parallel noch zeitmässig übereinstimmend ver-. laufen. Das spricht dafür, dass diese Quantitätsschwankungen von einem veränderlichen Faktor verursacht werden, der in Abhängigkeit von der aktuellen Situation grösseren oder kleineren Einfluss auf das Gefüge der Verbreitung der Hasen im Populationsareal hat. Als ein solcher Faktor könnten die klimatischen Verhältnisse angesehen werden.

Beachtenswert ist die ständige Verminderung der durchschnittlichen Siedlungsdichte von Jahr zu Jahr auf den waldnahen Feldern (Tabelle 3). Das könnte man mit der jedes Jahr kleiner werdenden Migrationsintensität der Hasen zwischen Wald und Feld in Verbindung bringen. In jedem Fall sind das parallele Erscheinungen und es ist durchaus möglich, dass sich bei starker Migrationsintensität ein grosser Teil der Hasen in der Nähe des Waldes ansiedelt.

In jedem Jahr gibt es einen Zeitabschnitt, in dem die Siedlungsdichte der Hasen auf allen Feldern (mit Ausnahme der dorfnahen Felder) beinahe gleich gross ist. Diese Moments wiederholen sich regulär in den einzelnen Untersuchungsjahren in der zweiten Septemberhälfte oder ersten Oktoberhälfte und in der zweiten Dezemberhälfte oder ersten Januarhälfte (Abb. 7a, b, c). Bisher fehlt eine Erklärung dieser Erscheinung. Die Regelmässigkeit ihres Auftretens spricht jedoch gegen reinen Zufall.

Es muss auf die Tatsache aufmerksam gemacht werden, dass die vier Varianten des Feldmilieus im Untersuchungsrevier eine grosse Mosaik bilden und dass ein jeder von ihnen in einem Komplex nur ausnahmsweise mehr als 100 ha umfasst. Umso bezeichnender ist die festgestellte Jifferenzierung der Siedlungsdichte der Hasen, die von einer starken Reaktion der Population auf die physischen Eigenschaften ihres Lebensraums zeugen kann.

\section{ORTSWAHL}

Wie im Abschnitt VI erwiesen wurde, ist die räumliche Verteilung der Hasenpopulation $u$. a. von einer Reihe Umweltselemente abhängig. Die dort durchgeführte Analyse hatte jedoch zum Ziel, die Veränderungen in der räumlichen Verteilung der ganzen Population oder grösserer Individuengruppen hervorzuheben, ohne auf die Situation der einzelnen Individuen oder kleiner Flächeneinheiten des gesamten Populationsareals näher einzugehen. Die gegenwärtige Erwägungen sollen auf einige, die Struktur der räumlichen Verteilung betreffende Momente, ein 
Licht werfen. Dank der Aufteilung des Taxationsstreifens auf $100 \mathrm{~m}$ lange Abschnitte, die Flächeneinheiten von $100 \times 100=1$ ha darstellen, ergab sich die Möglichkeit einer eingehenden Analyse gewisser Aspekte der Raumstruktur der untersuchten Population.

Analysiert man das zeitliche Vorkommen der Hasen auf den einzelnen Flächeneinheiten ${ }^{1}$ ), ist festzustellen, dass die Verteilung der Individuen im Gelände sehr ungleichmässig ist (Tabelle 4). Es gibt Stellen, wo die ganze Saison über nicht einmal ein Hase angetroffen wurde. Die meisten dieser Orte wurden übrigens von den Hasen in allen Jahren gemieden (z.B. die Abschnitte 1, 3, 7-9, 16, 17, 23, 32, 34, 39-43, 47, 49, 77-79, 87-88, 93-95). Umgekehrt gibt es aber auch Stellen, wo in der Regen Hasen vorkommen (z.B. auf den Abschnitten 2, 13, 24, 26-28, 53, 55-57, $59,62,64-65,74-75,83,90)$.

In Tabelle 4 ist eine Aufteilung der ganzen Taxationstrasse in Abschnitte, die durch die verschiedenen Milieuvarianten verlaufen, eingez.eichnet. Gekennzeichnet sind auch alle Flächeneinheiten mit minimaler Frequenz der Hasentreffen (bis zu 7 Treffen im Laufe der ganzen Untersuchungszeit, bei 46 möglichen Treffen), und alle Flächeneinheiten mit einer grösseren Frequenz als 18 Treffen. Diese Werte wurden aufgrund der berechneten Standartabweichung vom Mittelwert ermittelt. Die Standartabweichung ist bei einem 12,2 ausmachenden Mittelwert 5,55 gleich.

Ein Vergleich dieser beiden Werte ergibt, dass in Ubereinstimmung mit der im Abschnitt VI dieser Arbeit festgestellten Gesetzmässigkeit, die meisten Abschnitte der Trasse mit geringer Häufigkeitsfrequenz auf den sogenannten dorfnahen Feldern liegen. Jedoch auch in den anderen Biotopen des Feldmilieus kommen solche Orte vor. Die Abschnitte mit grosser Häufigkeitsfrequenz der Hasentreffen sind ebenfalls auf der ganzen Trasse zerstreut, wenn auch stellenweise deutliche Gruppierungen auftreten. Es muss hier bemerkt werden, dass so die Abschnitte des Taxationsstreifens, auf denen in der Regel Hasen anzutreffen waren, wie auch diejenigen, die von den Hasen gemieden wurden (mit Ausnahme der dorfnahen Felder), ansich durch nichts von Abschnitten mit mittlerer Häufigkeitsfrequenz zu unterscheiden sind. Vermutlich spielen hier schwer wahrnehmbare Elemente des Milieus eine Rolle, die an gewissen Orten optimale oder auch minimale Verhältnisse schaffen, durch welche die Art der Verteilung der Population im Areal bestimmt wird. Diese Elemente näher zu bezeichnen ist nicht einfach. Mittels der angewand-

1) Diese Analyse wurde für 2 ha grosse $(200 \times 100 \mathrm{~m})$ Flächeneinheiten durchgeführt. $\mathrm{Zu}$ diesem Zweck wurden die Zahlen zweier nebeneinander gelegenen $100 \mathrm{~m}$ langen Abschnitten des Taxationsstreifens summiert. Flächen von nur 1 ha Grösse erschienen bei der verhältnismässig geringen Gesamtdichte der Besiedlung. für eine derartige Analyse zu klein. 
ten Methoden „makro" waren sie nicht zu erfassen. Es ist möglich, dass es sich hier um mikroklimatische Elemente oder Bodenelemente handelt. Sehr wahrscheinlich ist auch die Möglichkeit, dass gewisse Faktoren des Milieus im scheinbar monotypischen Feldbictop soweit differenziert sind, dass dies seinen Widerholl im Unterschied der Dichte oder der Häufigkeitsfrequenz der Hasentreffen auf kleinen Geländeabschnitten findet. $\mathrm{Zu}$ solchen Faktoren könnte man den Bedrohungs- und Beunruhigungsgrad der Hasen zählen, der sogar auf kleinen, nebeneinander gelegenen Flächeneinheiten des Geländes unterschiedlich sein kann. Einen gewissen Einfluss auf diese Erscheinung hat bestimmt auch die schon oben beschriebene Präferenz der Hasen hinsichtlich der verschiedenen Feldkulturen. Insofern kann das keine entscheidende Bedeutung haben, da die entsprechenden Flächeneinheiten, bei der schon erwähnten Kleinackerwirtschaft auf diesem Gelände, immer eine ganze Reihe verschiedener Feldkulturen umfassen. Berücksichtigt werden müsste auch die durchaus mögliche Rolle der inneren Populationsverhältnisse, wie z.B. die Jahre hindurch an den selben Stellen auftretenden Gruppierungen von Hasen, die auf andere Hasen anziehend wirken können. Den ganzen mit der unregelmässigen Verbreitung der Individuen der Population im Areal verbundenen Komplex von Erscheinungen und Prozessen könnte man „Ortswahl” nennen. Die Ortswahl spielt gewiss so für die einzelnen Hasen, wie auch für die ganze Population eine bedeutsame Rolle.

Im Zusammenhang mit dem Problem der Ortswahl bei den Hasen wurde die Häufigkeitsfrequenz im zeitlichen Aspelst auf den einzelnen Flächeneinheiten analysiert. Bei dieser Analyse ist man aus der Voraussetzung hervorgegangen, dass das Gefüge der räumlichen Verteilung der Jasen dann gewisse Merkmale von Stabilität aufweist, wenn in mindestens zwei hintereinander folgenden Streifentaxationen die Zahl der Abschnitte, auf denen jedesmal Hasen angetroffen wurden grösser ist, als es aus reinem Zufall hervorgehen kann. Grössere Zeitabschnitte zu nehmen schien unzweckmässig, da dann das saisonale Aspekt der Verbreitung verwischt werden könnte.

Es wurde folgende Formel des Häufigkeitskoeffizients angewandt:

$$
\mathrm{S}=\frac{\mathrm{n} \cdot \mathrm{r}}{\mathrm{a} \cdot \mathrm{b}} \geqslant 1,5
$$

wo: $\mathrm{n}$ - Anzahl der Proben, $\mathrm{r}$ - Anzahl der Proben, bei denen in beiden analysierten Zeitabschnitten mindestens ein Hase angetroffen wurde, a - Anzahl der Proben, bei denen im ersten Zeitabschnitt mindestens ein Hase angetroffen wurde, b - Anzahl der Proben, bei denen im zweiten Zeitabschnitt mindestens ein Hase angetroffen wurde.

Ist der berechnete Koeffizient grösser als 1,5, dann ist die Häufigkeitsfrequenz grösser, als es bei reinem Zufall der Fall wäre. 
Die Tauglichkeit dieses Koeffizients für derartige Analysen wurde von Tarwid (1960) behandelt. Die Berechnungen wurden für Abschnitte der Taxationstrasse von $100 \times 100 \mathrm{~m}, 200 \times 100 \mathrm{~m}, 300 \times 100 \mathrm{~m}$ und $400 \times 100 \mathrm{~m}$ ausgeführt. Als Gesamtzahl der Proben wurde die aus der entsprechenden Aufteilung der Trasse hervorgehende Zahl der Abschnitte betrachtet.

Für Abschnitte von 300 und $400 \mathrm{~m}$ Länge hält sich der Koeffizient die ganze Saison über, also von September bis April der einzelnen Jahre in Grenzen unter 1,5. Bei 2 ha Flächengrösse der einzelnen Proben überschreitet er im Herbst die 1,5 Grenze. Bei 1 ha grossen Flächen ist er auch Ende Winter und im Vorfrühling real. Die Unterschiede in den einzelnen Jahren sind recht bedeutend, wobei der Verlauf der Kurven jedoch ähnlich ist (Abb. 8).

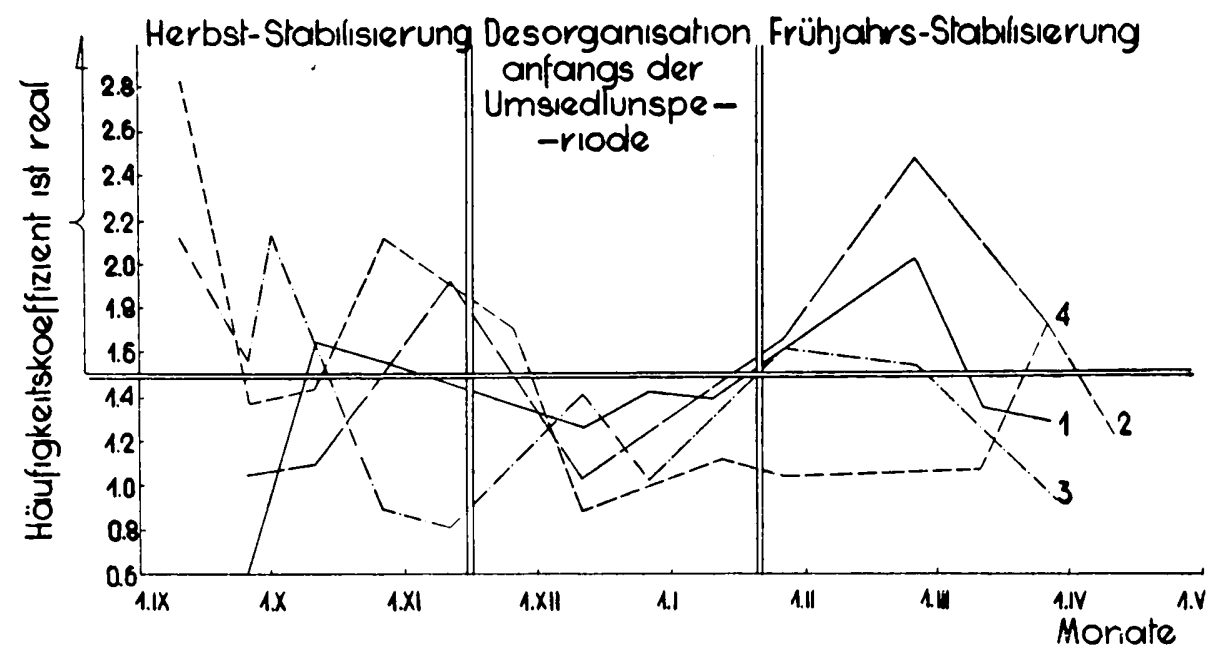

Abb. 8 Häufigkeitskoeffizient der Hasen auf den einzelnen $100 \mathrm{~m}$ Abschnitten des Taxationsstreifens. $1-1961 / 62,2-1962 / 63,3-1963 / 64,4-1964 / 65$.

Die Resultate dieser Analyse können auf zweierlei Wegen interpretiert werden. Der erste Weg das ist die Grösse der Flächeneinheiten. Wie die Berechnugen erwiesen haben, ist die Häufigkeitsfrequenz lediglich für ein und zwei Hektar grosse Flächeneinheiten real. Für grössere Flächen erreicht der Koeffizient nicht die 1,5 Grenze, wenn seine Schwankungen auch die gleiche Tendenz haben, wie bei kleineren Flächen. Das konnte davon zeugen, dass unabhängig von der Aufteilung des Populationsareals in verschiedene Milieuvariante, eine detaillierte Aufteilung des Geländes in kleine Abschnitte mit verschieden grosser Tauglichkeit für die Hasen besteht, die infolge der Einwirkung von Mikrofaktoren des Milieus beziehungsweise auch unter dem Einfluss der Alstivität der Ha- 


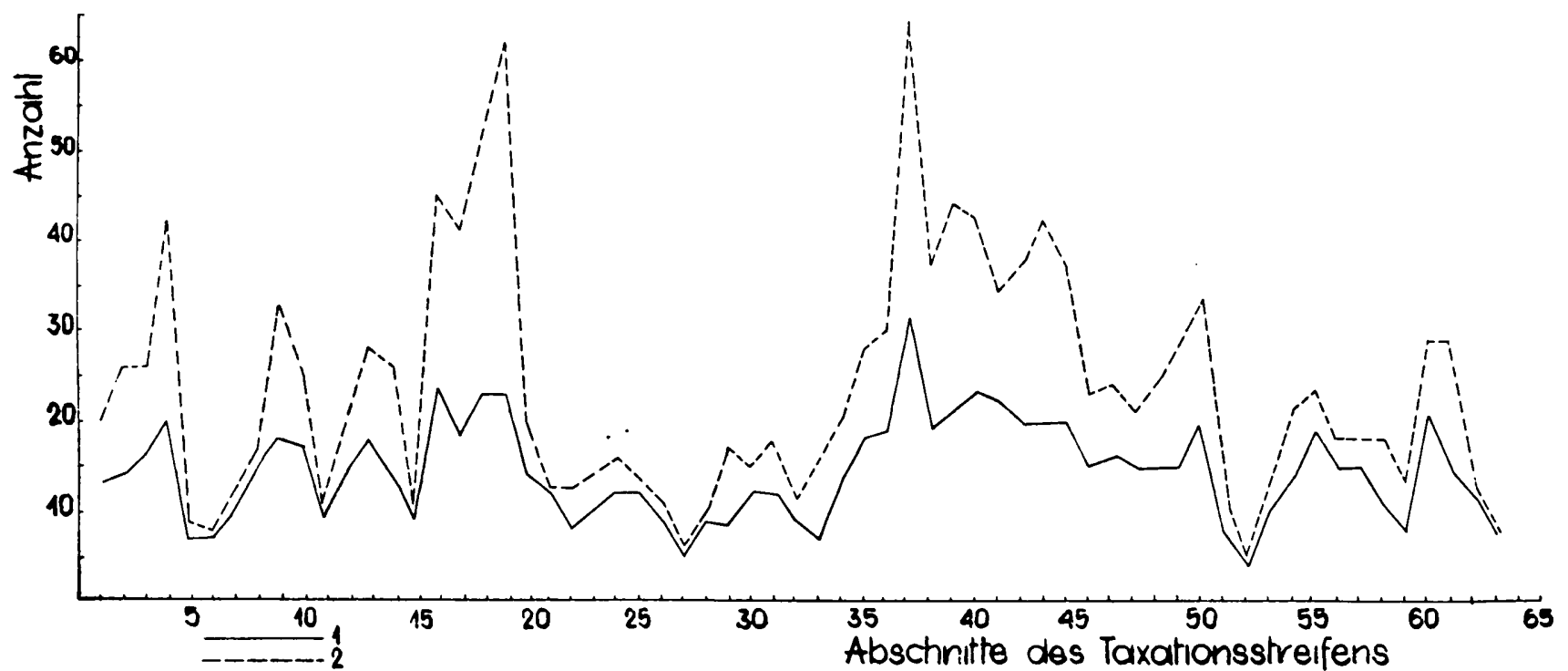

Abb. 9. Häufigkeit der Hasen auf den einzelnen Abschnitten des Taxationsstreifens. 1 - Anzahl der Hasentreffen, 2 - Anzahl der angetroffenen Individuen. 
sen als solche, entstanden ist. Diese Tatsache kann als Bestätigung der schon formulierten Vermutung gelten, dass die vorstehend beschriebene Ortswahl der Hasen in der Tat sehr kleine Geländeabschnitte betrifft.

Der zweite Weg, dass ist die Realität des Häufigkeitskoeffizients im Herbst und Ende Winter und dessen Realitätsmangel zur übrigen Zeit. Es kann sein, dass dies mit der festgestellten Zunahme der Siedlungsdichte verbunden ist. Im Herbst, wenn keine Migrationsbewegungen stattfinden, ist die Verteilung der Hasen im Revier stabil. Die dann später eintreffenden Migranten können wohl eine gewisse Desorganisierung im Raumgefüge herbeiführen, das sich erst nach einer gewissen Zeit wieder stabilisiert (Abb. 8).

\section{GRUPPIERUNGSTENDENZ}

Ein Vergleich der Anzahl an Hąsentreffen insgesamt auf den einzelnen Flächeneinheiten mit der Anzahl angetroffener Individuen weist darauf hin, dass im Falle einer geringen Anzahl von Treffen auch die Anzahl an Individuen, aus denen diese Treffen bestehen, nicht viel grösser ist. An Orten häufigen Vorkommens von Hasen ist die Anzahl an Individuen unverhältnismässig hoch (Abb. 9). Die Dichte je Flächeneinheit ist hier bedeutend grösser als an anderen Stellen. Es besteht also eine gegenseitige Abhängigkeit zwischen der Ortswahl und dem Entstehen von Gruppierungen an bestimmten Orten.

Mit Hilfe des Testes $t$-Student wurde die Realität des Variabilitätskoeffizients $V$ geprüft, der für die erhaltenen Verteilungsfunktionen der Hasen als Gruppierungskoeffizient gelten kann. Ist das berechnete $t$ kleiner als $t_{0,05}=2,000$, dann heisst das, dass die Verteilung der Hasen im analysierten Zeitabschnitt eine zufällige war. Ist $t$ grösser als 2,000, dann ist die Verteilung nicht zufällig. Tabelle 5 enthält eine Aufstelllung des berechneten $t$ für 1, 2 und 3 ha grosse Einheiten der taxierten Fläche. Ein Vergleich der Ergebnisse dieser Berechnungen lässt erkennen, dass Zeitabschnitte vorkommen, wo unabhängig von der angewandten Grösse der Flächenprobe, der Koeffizient nicht real ist, wo also die Verteilung eine Sache des Zufalls ist. Für die Herbstzeit lässt sich diese Tatsache durch die geringe Siedlungsdichte im gesamten Untersuchungsrevier erklären. In anderen Zeitabschnitten der Saison jedoch, wie z. B. in der Ir. Märzhälfte 1961, II. Februarhälfte 1962, II. Märzhälfte 1963, I. Januarhälfte 1964 und II. Februarhälfte 1965 handelt es sich klar um zeitweisen Schwund der Gruppierungstendenz bei den Hasen, aus schwer erklärbaren Ursachen. Es kann sein, dass diese Erscheinung irgendwie mit dem Vermehrungszyklus (Paarungszeit, Setzzeit usw.) verbunden ist.

In den einzelnen Jahren ist eine gewisse Abhängigkeit der Gruppierungstendenz und der Häufigkeitsfrequenz ersichtlich. Die beide Er- 
Tabelle 5.

Variabilitätskoeffizient $V$, geprüft mit dem Test $t$-Student.

\begin{tabular}{|c|c|c|c|c|c|c|c|c|c|c|c|c|c|c|c|}
\hline \multirow{2}{*}{ 2eitabschnitt } & \multicolumn{3}{|c|}{$1961 / 62$} & \multicolumn{3}{|c|}{$1961 / 62$} & \multicolumn{3}{|c|}{$1962 / 63$} & \multicolumn{3}{|c|}{$1963 / 64$} & \multicolumn{3}{|c|}{$1964 / 65$} \\
\hline & $\begin{array}{l}\text { für } \\
1 \text { ha }\end{array}$ & $\begin{array}{l}\text { rür } \\
2 \text { ha }\end{array}$ & $\begin{array}{l}\text { sur } \\
3 \text { ha }\end{array}$ & $\begin{array}{l}\text { eür } \\
1 \text { ha }\end{array}$ & $\begin{aligned} & \text { für } \\
2 & \text { na }\end{aligned}$ & $\begin{array}{l}\text { für } \\
3 \text { ho }\end{array}$ & $\begin{array}{l}\text { für } \\
1 \text { ha }\end{array}$ & $\begin{array}{l}\text { für } \\
2 \text { tra }\end{array}$ & $\begin{array}{l}\text { für } \\
3 \text { ha }\end{array}$ & $\begin{array}{l}\text { für } \\
\text { i ha }\end{array}$ & $\begin{array}{l}\text { für } \\
2 \text { ha }\end{array}$ & $\begin{array}{l}\text { für } \\
3 \text { ha }\end{array}$ & $\begin{array}{l}\text { für } \\
1 \text { ha }\end{array}$ & $\begin{array}{l}\text { für } \\
2 \text { ha }\end{array}$ & $\begin{array}{r}\text { für } \\
3 \text { ha }\end{array}$ \\
\hline $1-15 I X$ & & & $\therefore$ & - & - & - & 0,97 & 1,38 & 0,39 & 3,79 & 1,93 & 1,19 & - & - & - \\
\hline $16-30 I X$ & & & & 0,87 & 1,24 & 0,67 & 1,84 & 1,32 & 2,01 & 0,58 & 0,00 & 1,17 & 0,00 & 0,00 & 0,00 \\
\hline $1-15 x$ & & & & 0,68 & 0,00 & 0,57 & 0,97 & 0,69 & 1,84 & 0,00 & 0,69 & 0,34 & 0,00 & 0,96 & 1,39 \\
\hline $16-31 x$ & & & & - & - & - & 2,33 & 1,03 & 3,13 & 3,79 & 5,38 & 4,47 & - & - & - \\
\hline $1-15 X I$ & & & & 0,58 & 0,21 & 0,56 & - & - & - & - & - & - & 4,85 & 1,03 & 1,67 \\
\hline $15-j 0 X I$ & & & & - & - & - & 3,79 & 3,17 & 3,18 & 3,69 & 3,52 & 3,69 & - & - & - \\
\hline $1-15 X I I$ & & & & 1,08 & 0,97 & 1,01 & 1,84 & 3,52 & 2,63 & 2,33 & 3,17 & 2,23 & 0,00 & 0,69 & 0,56 \\
\hline $15-31 X I I$ & & & & 1,75 & 4,98 & 6,48 & - & - & - & - & - & - & - & - & - \\
\hline $1-15 I$ & & & & 4,39 & 2,21 & 1,67 & 2,82 & 3,45 & 2,85 & 0,58 & 0,55 & 0,34 & 1,55 & 3,10 & 1,67 \\
\hline $16-31 I$ & & & & - & - & - & 2,62 & 3,86 & 3,52 & 3,69 & 5,86 & 7,15 & - & - & - \\
\hline $1-15 \mathrm{II}$ & 2,23 & 1,24 & 1,73 & - & - & - & - & - & - & - & - & - & - & - & - \\
\hline $16-28 I I$ & $0,7 \varepsilon$ & 1,17 & 2,57 & 0,68 & 0,21 & 0,73 & - & - & - & 5,73 & 2,55 & 3,35 & $0,1 t$ & 1,17 & 1,01 \\
\hline $1-15 \mathrm{III}$ & 1,08 & 2,97 & 1,79 & 4,65 & 4,79 & $7,60^{\circ}$ & 2,04 & $\cdot 2,03$ & 4,58 & - & - & - & - & - & $\therefore$ \\
\hline $16-31$ III & 0,29 & 0,00 & 0,73 & 7,57 & 4,28 & 5,79 & 1,55 & $0, \subseteq 0$ & 1,63 & 2,72 & 4,75 & 3,52 & 2,91 & 1,61 & 2,06 \\
\hline$i-15 I V$ & 1,26 & 3,38 & 2,23 & 0,29 & 5,31 & 1,28 & 4,85 & 4,34 & $2, \subseteq 6$ & - & - & - & - & - & - \\
\hline $15-30 \mathrm{IV}$ & - & - & - & - & - & $\cdot$ & 2,82 & 4,73 & 3,07 & 1,94 & 1,93 & 3,30 & 2,42 & 4,28 & 1,67 \\
\hline
\end{tabular}


scheinungen charakterisierenden Koeffizienten weisen entgegengesetzte T'endenz auf. Ist der Häufigkeitskoeffizient real, dann ist der Gruppierungskoeffizient nicht real und umgekehrt (Abb. 10). Diese Abhängigkeiten trifft zwar nicht in allen Fällen zu, aber beide Erscheinungen sind schliesslich nicht nur ausschliesslich unmittelbar voneinander ab-
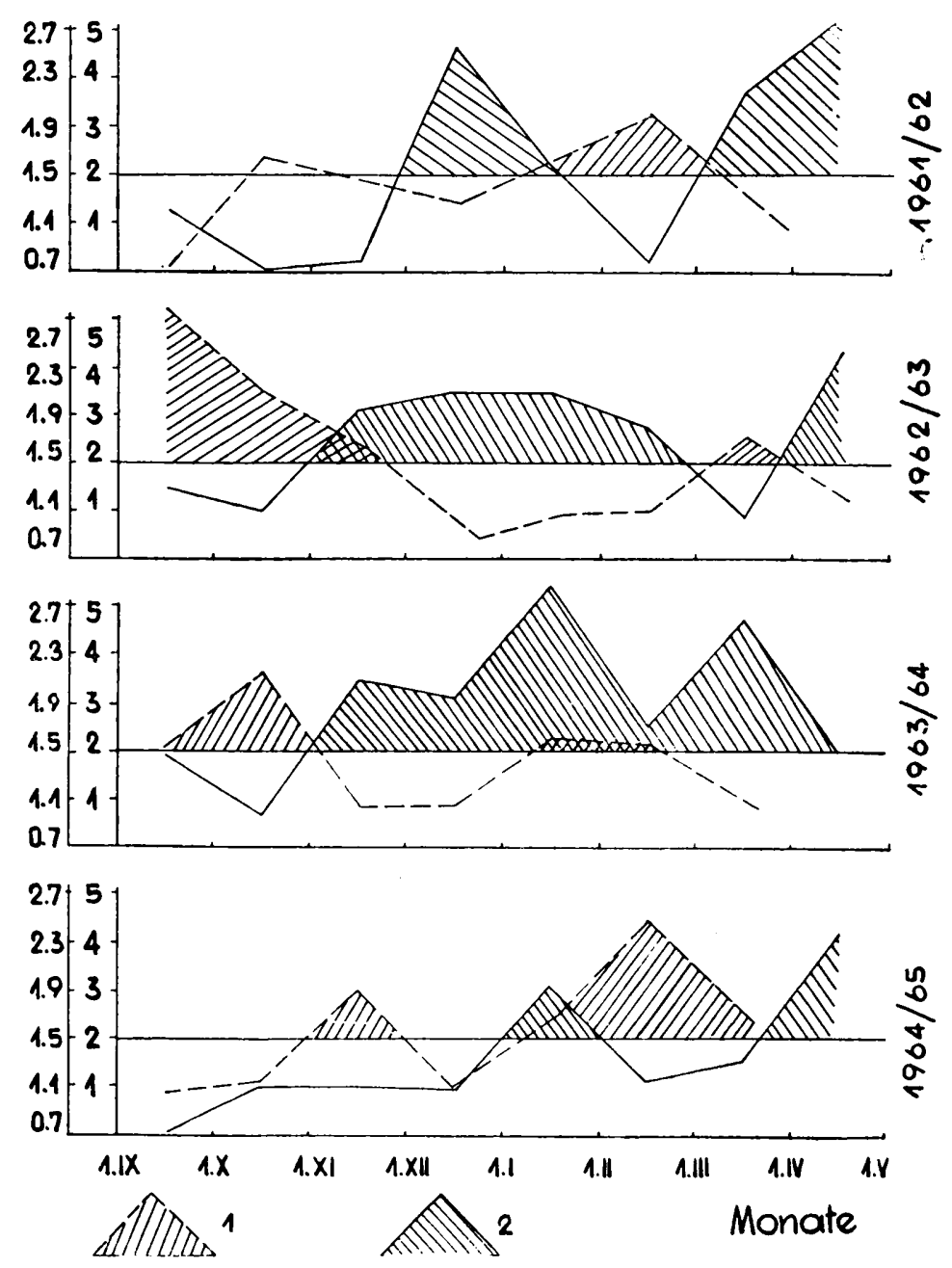

Abb. 10. Häufigkeitskoeffizient und Gruppierungskoeffizient, in monatlichen Zeitabschnitten zusammengestellt. 1 - Häufigkeit ist real, 2-Gruppierungen sind real.

hängig. Dennoch hat es den Anschein, dass hier eine gewisse Gesetzmässigkeit auftritt. Bei Zunahme der Gruppierungstendenz verringert sich die Zahl der positiven Proben, d. h. die Zahl der Abschnitte, auf denen Hasen angetroffen wurden. Gleichzeitig vermindert sich die 
Chance, in zwei nacheinander folgenden Taxationen Hasen auf den selben Abschnitten der Trasse anzutreffen, wodurch natürlich der Häufigkeitskoeffizient unreal wird.

Ein weiteres Problem ist die Frage der Grösse der entstandenen Hasengruppierungen. Als Gruppierung wurden solche Situationen angesehen, wenn wenigstens zwei Individuen gleichzeitig auf der gleichen Flächeneinheit ( $2 \mathrm{ha}$ ) vorkamen. Dabei wurde vorausgesetzt, dass Hasen die sich auf diesen zwei Hektaren befinden, untereinander Hör-, Geruch-

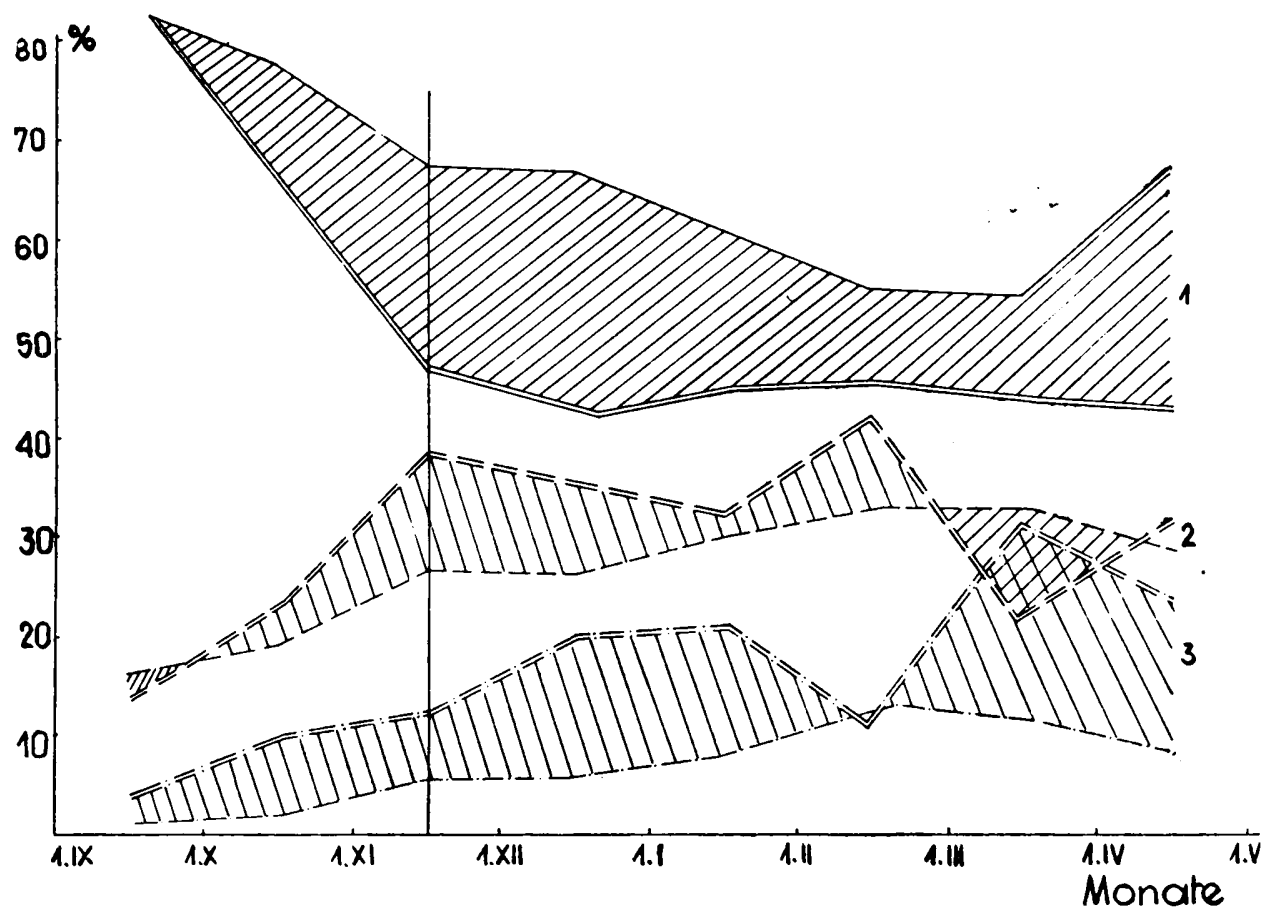

Abb. 11. Hasengruppierungen auf den einzelnen Abschnitten des Taxationsstreifens (Flächen von 2 ha Grösse) in monatlichen Zeitabschnitten insgesamt für die Jahre 1961-1964. 1 - ein Hase, 2 - zwei Hasen, 3 mehr als zwei Hasen. Durch Doppellinien werden die empirischen Funktionen, durch einfache Linien - die theoretischen Funktionen bezeichnet.

oder Sehkontakt haben und dass ihre gegenseitige Annäherung nicht eine Frage des Zufalls ist. Ein solches Kriterium annehmend stützte man sich $u$. a. auf vielen Beobachtungen die davon zeugen, dass der Kontaktgrad zwischen Individuen einer so aufgefassten Gruppierung bedeutend grösser ist, als zwischen anderen Hasen. Das Hochmachen eines Hasen aus der Sasse verursacht z. B. ein gleichzeitiges Hochwerden auch der übrigen Individuen der Gruppierung. 
Die Grössefunktionen der Grupierungen wurden monatlich für die ganze Untersuchungszeit zusammen analysiert. Ab November hebt sich ein bestimmtes Grössengefüge der Gruppierungen ab, dass bei geringen Abweichungen dann schon bis zum Frühjahr anhält (Abb. 11). In den Monaten September und Oktober ergab sich ein anderes Gefüge, dass aber Resultat der Untersuchungsmethode sein kann, d.h. Ungenauigkeit der Streifentaxation zu dieser Zeit, und somit bei der Analyse nicht berücksichtigt werden konnte. Im März und April vergrösserte sich der Anteil mehrstückiger Gruppierungen, jedoch nicht auf Kosten der einzelnen Hasen, was zu erwarten war, sondern auf Kosten der zweistückigen Gruppierungen, deren Anzahl sich verringerte. Die Gruppierungen oder auch der von ihnen eingenommene Platz wirkt also anziehend. Bezeichnend ist der Anteil einzelner Hasen, der sich von November bis zum

Tabelle 6.

Gruppierungstendenz der Hasen, geprüft mittels der Statistik $\chi^{2}$, insgesamt für 4 Untersuchungsjahre.

\begin{tabular}{|l|c|c|c|}
\hline Monat & $\begin{array}{c}x^{2} \\
\text { berecnnet }\end{array}$ & $<$ & $\begin{array}{c}x^{2} \\
\text { theoretisch }\end{array}$ \\
\hline September & 0,04 & 11,342 \\
Oktober & 3,93 & $<$ & 11,345 \\
November & 12,73 & $>$ & 11,345 \\
Dezember & 23,57 & $>$ & 11,345 \\
Januar & 18,73 & $>$ & 13,277 \\
Februar & 8,54 & 13,277 \\
März & 49,61 & $>$ & 13,277 \\
April & 28,49 & 13,277 \\
\hline
\end{tabular}

F'ühjahr unveränderlich auf einem Niveau von ca $45 \%$ hält. Die Abweichung von der theoretischen Poisson-Funktion ist bedeutend. Auch die Kurven der Hasengruppierungen haben einen anderen Verlauf, als die theoretischen Kurven (Abb. 11). Die Realität der Abweichungen der empirischen Daten von den theoretischen Daten nach der Wahrscheinlichkeit in der Poisson-Funktion wurde mittels der $\chi^{2}$ Statistik geprüft. 
Dabei erwies sich, dass die Unterschiede im November, Dezember, Januar, März und April wesentlich sind, in den übrigen Monaten jedoch nicht (Tab. 6). Der Realitätsmangel der Unterschiede im September und Oktober kann analogisch auch ein Ergebnis der Methodik sein.

Die Erscheinung einer gewissen Stabilität des Grössengefüges der Gruppierungen in Zusammenstellung mit der festgestellten Gruppierungstendenz könnte davon zeugen, dass die Population unabhängig von der Dichte, die in diesem Fall laufend zunimmt, einen ständiges, den Gruppierungsgrad bestimmendes Raumgefüge besitzt.

\section{DISKUSSION DER ERGEBNISSE}

Die erzielten Untersuchungsergebnisse erlauben es auf einige Elemente der Raumstruktur hinzuweisen, durch welche die untersuchte Hasenpopulation charakterisiert wird. $\mathrm{Zu}$ ihnen gehören:

- saisonale Dichteveränderungen im Feldrevier, die infolge regulärer Wanderungen eines Teils der Individuen innerhalb des Populationsareals entstehen,

- Unterschiedlichkeit der Siedlungsdichte in den verschiedenen Varianten des Feldmilieus,

- unterschiedliche Häufigkeitsfrequenz der Hasen auf Flächeneinheiten von geringer Grösse,

- gruppierte Verteilung der Hasen im Populationsareal.

Es ist schwer zu sagen, wie weit diese Elemente typisch für die Raumverteilung der Hasenpopulationen überhaupt sind, denn es mangelt an Vergleichsmaterial aus anderen Gebieten. Eine Reihe von Momenten in dieser Arbeit scheint jedoch darauf hinzuweisen, dass sich die unmittelbar von der ökologischen Situation im gegebenen Revier abhängende Raumstruktur der Hasenpopulationen in bestimmten ökologischen Verhältnissen bildet und Ausdruck einer Adaptation der Population an diese Verhältnisse ist. Das wäre eine Bestätigung der Anschauung von Petruse wicz (1966).

Versuchen wir dieses Problem etwas näher zu analisieren. Wie schon erwähnt, sind die im Untersuchungsgebiet herrschenden ökologischen Verhältnisse allem Anschein nach für die Hasen ausnehmend ungünstig (siehe Geländebeschreibung), haben jedoch noch nicht den kritischen Minimalstand erreicht, unter welchem das Bestehen der Population in einer solchen Dichte, die ihr volles Weiterbestehen garantiert - überhaupt nich möglich ist. Ubereinstimmend mit der vorstehend ausgesprochenen These führt das Streben der Population nach gedeihlichen Weiterbestehen in ungünstigen ökologischen Verhältnissen zu einer Reihe 
von Adaptationen, die in der Spezifik der ökologischen Organisation der Population, also u. a. auch in der Raumstruktur ihren Ausdruck finden.

In der untersuchten Population kann man eine Reihe von Anzeichen dieser Anpassungen finden, z.B. die in der Population stattfindenden, durch zeitweise Veränderungen des Fassungsvermögens des Lebensraums verursachte saisonale Migrationen eines Teils der Hasen. Die aktive Wirkung einer Reihe äusserer Faktoren vergrössert zu gewissen Jahreszeiten stark den Gefahren- und Beunruhigungsrad der Hasen, wodurch sich das effektive Fassungsvermögen des Feldmilieus verkleinert. Der gesamte Überschuss an Individuen ist gezwungen auszuwandern, um zu gegebener Zeit wieder auf die Felder zurückzukehren. Dank dieser regulären Umsiedlung kann sich die Gesamtdichte der Population auf einem bedeutend höheren Niveau erhalten, als es anders möglich wäre, was für das Los der Population gewiss günstig ist.

Als weiteres Zeichen des modifizierenden Einflusses der äusseren Faktoren auf die Raumstruktur kann man die Differenzierung der Siedlungsdichte in den verschiedenen Varianten des Feldmilieus ansehen. Die dorfnahen Felder werden gemieden, auf anderen Feldtypen kommen starke quantative Fluktuationen vor. In jedem Jahr wiederholen sich Zeitabschnitte, in denen sich die Siedlungsdichte im ganzen Revier ausgleicht. Es ist kaum anzunehmen, dass alle diese Erscheinungen reiner Zufall sind, wenn ihr Sinn auch nicht immer klar ist. Wenn noch in Betracht gezogen wird, dass die vier Milieuvarianten im Untersuchungsgebiet eine Mosaik aus kleinen Flächen bilden, dann wird die festgestellte Unterschiedlichkeit der Siedlungsdichte im Raum noch mehr bezeichnender. Sie zeugt von einer starken Reaktion der Population auf die physischen Eigenschaften des Lebensraums, in dem die existiert.

$\mathrm{Zu}$ derselben Kategorie von Erscheinungen gehört auch die unterschiedliche Häufigkeitsfrequenz der Hasen auf ganz kleinen Flächen, die vermutlich infolge einer detaillierten Differenzierung des Geländes in verschieden auf die Hasen anziehend wirkende Abschnitte entstanden ist. Die Population reagiert sehr feinfühlig auf die Differenzierung des Lebensraums und schafft den einzelnen Individuen durch die Anpassung der räumlichen Verteilung an die aktuelle Umweltssituation grössere Überlebungschancen.

Alle diese Erscheinungen bewahrten die Population jedoch nicht vor ciner grossen Bestandsabnahme in den letzten Jahren. Die Durchschnittsdichte pro Flächeneinheit des Populationsareals verkleinerte sich deutlich und weist dauernde Tendenz eines weiteren Gefälles auf. Die Reduktion des Bestandes durch Jagdschädlinge (Hunde, Katzen, Krähen usw.) ist bei mangel an Schutz, Winterfütterung und dergleichen von Seiten des Menschen, derart stark, dass die Quantität laufend geringer 
wird. Nimmt man die These von Petruse wi c z (1966) auf, dass die Dichte der einfachste aber sehr wesentliche Ausdruck der Raumstruktur ist, könnte man behaupten, dass bei den im Untersuchungsgebiet herrschenden Verhältnissen, bei alljährlich sich wiederholenden Zeitabschnitten, in denen die Hasen besonders stark Gefahren ausgesetzt sind, eine geringere Siedlungsdicht für die einzelnen Individuen günstiger sein kann. Die saisonalen Umsiedlungen, durch welche die Dichte auf den Feldern dem aktuellen Fassungsvermögen entsprechend, reguliert wird, weisen darauf hin, dass es in der Tat der Fall ist. Der Migrationsprozess hat jedoch keinen Einfluss auf die Verminderung der Gesamtquantität der Population und ist somit vom Gesichtspunkt der Population aus gesehen, von der die Erhaltung einer bestimmten Bestandsgrösse bei ungünstigen Verhältnissen bestrebt wird, durchaus verständlich. Dennoch wird die Quantität laufend geringer und die festgestellten Adaptationserscheinungen der Raumstruktur an diese Verhältnisse, können lediglich das Quantitätsgefälle etwas hemmen, haben jedoch keinen entscheidenden Einfluss.

In solcher Situation wird eine starke Ingerenz des Menschen, in der jagdlichen Terminologie als „Hegemassnahmen” bekannt, deren Zweck eine Verbesserung der Lebensbedingungen des Wildes ist, absolut notwendig. Es muss hier eine bisher noch nicht erwähnte Tatsache unterstrichen werden, dass es eine methodische Voraussetzung dieser Arbeit war, im Untersuchungsgebiet keine jagdlichen Hege- und Schutzmassnahmen durchzuführen. Die nächste mehrjährige Forschungsetappe sieht eine grosse Intensifikation verschiedener Hegemassnahmen vor, deren Einfluss auf das weitere Los der Hasenpopulation dann beobachtet werden soll.

Wie schon in der Einführung erwähnt wurde, kommen solche Gebiete wie das Untersuchungsrevier, in Polen verhältnismässig häufig vor, wobei die schnelle Entwicklung der Urbanisierung des Landes darauf hinweist, dass ihrer immer mehr werden wird. Das Verfolgen des Einflusses der Umweltsverenderungen auf das hier lebende Wild muss also zu den Forschungsthemen der jagdlichen Forschungsstellen gehören. Die vorliegende Arbeit, die lediglich ein Aspekt dieses sehr komplizierten Problems behandelt, stellt kaum einem Anfang eines ganzen Forschungskomplexes dar, der dieses Problem betreffen müsste. Es ist sehr wahrscheinlich, dass schon in nicht weiter Zukunft die Frage der Erhaltung des Wildes auf urbanisierten, dicht bewohnten und verschiedenartig vom Menschen ausgebauteten Gebieten - und das Problem einer regulären .Jagdwirschaft in diesen Verhältnissen, ein immer grösser werdendes Gewicht haben wird. 


\section{SCHLUSSFOLGERUNGEN FUR DIE PRAXIS}

Die vorliegenden Untersuchungen waren nicht darauf eingestellt, Resultate zu erzielen, die unmittelbar zu völligen Lösung eines konkreten Problems in der Jagdwirtschaft beitragen sollten. Sie waren mehr auf gewisse theoretische ökologische Probleme eingestellt, die erst in der weiteren Perspektive Anwendung in der Jagdpraxis finden können. Da jedoch der Bedarf nach durch Forschungsergebnissen gestützten praktischen Hinweisen schon gegenwärtig sehr gross ist, erscheint es zweckmässig, gewisse praktische Schlussfolgerungen aus Forschungsarbeiten über den Hasen sogar schon dann zu formulieren, wenn sie gezwungerweise auch nur begrenzte Reichweite oder Charakter kleiner Beiträge haben können.

Aus solcher Voraussetzung hervorgehend, wurde verursacht, aufgrund des in der vorliegender Arbeit enthaltenen Materials einige praktische Hinweise $\mathrm{zu}$ formulieren, die schon jetzt in Revieren von ähnlichem Charakter wie das Forschungsrevier, bei der Einrichtung der Jagdwirtschaft hinsichtlich des Feldhasen behilflich sein können.

1. Die festgestellten regulären saisonalen Dichtefluktationen der Hasenpopulation im Untersuchungsgebiet sind nachdrückliche Illustrierung dessen, dass die den Jagdgesellschaften zur allgemeinen Awendung emplohlenen Streifentaxationen der Hasen, als quantitative Methode nur dann ein der Wirklichkeit nahes Quantitätsbild geben, wenn sie mehrmals in der Saison wiederholt werden. Man kann aber noch weiter gehen und aufgrund des vorliegenden Beispiels behaupten, dass die in vielen Jagdgesellschaften nur einmal im Jahr, ganz gleich mit welcher Methode durchgeführte Bestandsaufnahme der Hasen, ein völlig falsches Bild geben kann. Würde man im Untersuchungsrevier z.B. nur aufgrund der Frühjahrs-Bestandsaufnahme über den Hasenbestand folgern, könnte man den Stand als mindestens mittelmässig bezeichnen. Im nächsten Jahr würde sich dann während der Jagdsaison erweisen, dass nur sehr wenig Hasen da sind, was im Jahresdurchschnitt wiederum auch nicht mit der Wirklichkeit übereinstimmt.

2. In bestimmten Reviersituationen ist die jagdliche Nutzung nicht der entscheidend auf die Bestandshöhe einflusshabende Faktor. Wie schon crwähnt, wurde im Forschungsrevier eine Reihe von Jahren hindurch überhaupt nicht gejagd. Dennoch verringerte sich der Zahlenbestand der Hasenpopulation von Jahr zu Jahr. Eine normale jagdliche Nutzung des Reviers würde bei der zur Jagdzeit bestehenden Siedlungsdichte von etwa 20 Hasen pro 100 ha und bei dem in Polen angewandten Jagdsystem (jedes Jahr wird nur eine Hälfte des Reviers bejagd und das ausschliesslich in Vorstehtreiben) im Ergebnis wahrscheinlich eine Jahres- 
strecke von nicht mehr als $70-80$ Hasen geben, was ungefähr $10 \%$ des Giesamtbestands der Population ausmacht.

$\mathrm{Da}$ die natürliche Wintereduktion bis zu $50 \%$ des spätherbstlichen Bestands betrifft, also entschieden grösser ist als die jagdliche Nutzung, ist es augenscheinlich, dass die Jagd in dieser Situation keinen grossen Einfluss auf die Quantitätsschwankungen haben kann. In solchem Falle ist es also zwecklos die Jagd einzustellen, um den Bestand höher zu bringen.

3. Die Kenntnis der Tatsache, dass im Revier grössere saisonale Hasenwanderungen stattfinden, kann behilflich dabei sein, die jagdliche Nutzung des Bestands richtig durchzuführen. Im Untersuchungsrevier würde eine Jagd zum Anfang der Saison nur sehr schlechte Ergebnisse geben, die in keinem Verhältnis zur wirklichen Höhe des Bestands stehen. Würde wiederum erst im Wald gejagt werden und dann später noch auf den Feldern, dann würde der Bestand de facto zweimal bejagd werden, was eine übermässige Exploatation wäre. Bei gewissen Revierverhältnissen muss also von dem steifēn Grundsatz einer nur einmaligen Bejagung des Reviers abgesehen werden.

4. Die im Ergebnis der vorliegenden Untersuchungen festgestellte Präferenz bezüglich zu Feldkulturen, die möglichst gute Deckung geben, unterstreicht die Wichtigkeit des von vielen Verfassern aufgenommenen Problems, den Hasen im Felde verschiedenartige natürliche Deckung zu schaffen. Weiter konnte festgestellt werden, dass das ganze Revier in relativ kleine Abschnitte von verschieden grosser Tauglichkeit für den Hasen aufgeteilt ist. Wenn auf eine grössere oder kleinere Anziehungskraft des gegebenen Platzes im Revier auf die Hasen auch die Anwesenheit günstiger Deckung Einfluss hat, kann dadurch, dass möglichst viel solcher Deckung geschaft wird, die Chance einer Ansiedlung von Hasen auch an Orten, die aus anderen Gründen nicht dazu geeignet sind, beträchtlich vergrössert werden. Es vergrössert sich das gesamte Fassungsvermögen des Reviers und damit entstehen neue Möglichkeiten zur Hebung des Zahlenbestands der Population.

Danksagung: Es ist meine angenehme Pflicht, dem Direktor des Ökologischen Instituts, Herrn Prof. Dr. K. P e tr u s e wi c z, unter dessen Leitung die vorliegende Arbeit ausgeführt wurde, herzlichst für wertvolle Hinweise bei der Einsammlung und Bearbeitung des Materials zu danken. Mein Dank gilt auch den Herren Dr. R. Andrzejewski und Mgr. W. Jezierski, die sich der Mühe unterzogen, die kritische Durchsicht des Manuskript durchzuführen.

\section{SCHRIFTTUM}

1. Allee W. C., Emerson A. E., Park O., Park T., Schmidt K. P., 1958: Zasady ekologii zwierząt. Państw. Wyd. Nauk. 1: 1-598. Warszawa.

2. Andrzejewski R. \& Jezierski, W:, 1966: Studies on the European hare. XI. Estimation of population density and attempt to plan the yearly take of hares. Acta theriol., 11, 21: 433-448. 
3. B oback A. W., 1957: Der Feldhase und seine Hege. Merkblatt 4. Arbeitsgemeinschaft für Jagd- und Wildforschung. Deutsche Akad. der Landwirtschaftswiss. Berlin: 1-28.

4. Dobrowolski K., Pielowski Z., Pinowski J. \& Wasilewski A., 1958: Einfiuss der Änderung in der Biologie des Raben (Corvus corax L.) Brüten in nächster Nähe des Menschen - auf Grösse und Verteilung der Population dieser Vogelart. Ekol. pol., Ser. A, 6, 5: 167-182: Warszawa.

5. Hegendorf 1933: Der Hase, dessen Hege und Jagd. Richter u. Springer: $1-265$. Wien.

6. Jezierski W. \& Pielowski Z., 1958: Badania ankietowe nad zającem. Łowiec pol. 21 (1096): 3-4. Warszawa.

7. K o e n e n F., 1956: Der Feldhase. Die Neue Brehm-Bücherei 169: 1-80. A. Ziemsen Verlag. Wittemberg-Lutherstadt.

8. K ok e š O., 1948: Zajic: 1-153. Praha.

9. N a u m ov N. P., 1961: Ekologia zwierząt. Państw. Wyd. Roln. i Leśne: 1-569. Warszawa.

10. Odum E., 1963: Podstawy ekologii. Państw. Wyd. Roln. i Leśne: 1-560. Warszawa.

11. Petrusewicz K., 1966: Dynamics, organization and ecological structure of population. Ekol. pol., Ser. A 14, 25: 413-436. Warszawa.

12. M üller-Using D., 1962: Diezels Niederjagd. Verlag Paul Parey: 1-367. Berlin.

13. P i e low ski Z., 1961: Uber der Unifikationseinfluss der selektiven Nahrungswahl des Habichts (Accipiter gentilis L.) auf Haustauben. Ekol. pol., Ser. A 9, 11: 183-194. Warszawa.

14. Pielowski Z., 1962: Uber ökologische Forschungen am Feldhasen in Polen. Tagungsber., 55. Beitr. zur Jagd- und Wildforschg., 2: 41-52. Berlin.

15. Pin ow ski J., 1959: Factors influencing the number of feeding rooks (Corvus frugilegus frugilegus L.) in various field environments. Ekol. pol., Ser. A 7, 16: 435-482. Warszawa.

16. Pinowski J. \& Wasilewski A., 1962: Einfluss einiger Faktoren auf die Zahl der Nebelkrähen (Corvus corone cornix L.) in verschiedenen Biotopen. Acta ornithol., 6, 14: 231-251. Warszawa.

17. R a c z ński J., 1964: Studies on the European Hare. V. Reproduction. Acta theriol., 9, 19: 305-352.

13. Rieck W., 1956: Untersuchungen über die Vermehrung des Feldhasen. Zeitschr. Jagdwiss., 2, 2: 49-90. Berlin-Hamburg.

19. Rieck W., 1963: Der Feldhase. Merkblätter des Niederwildausschusses des DJV Nr. 4: 1-56. F. C. Mayer Verlag. Müchen.

20. Ruskov M. \& Petrov P. M., 1957: Proučvania vrhu niakoi ot mietoditie za taksiranie na zapasa ot zajci $i$ ustanoviavanie na najpodhodiaščitie za nasata praktika. Naučni trudovie, 5: 177-217. Minist. na Ziemied. i Goritie. Sofia.

21. Scarlatescu G. H., Almasan M. \& Nestorov V., 1963: Contributii la Cunoasterea Biologiei Iepurelui in R.P.R. Studii si Cercetari, 23-A: 97-115. Bucuresti.

22. Szederjei A., 1958: Z węgierskich badań nad zającem. Łowiec pol., 20 (1095): 2-3. Warszawa.

23. Szederjei M., Szederjei A. \& Studinka L., 1959: Hasen, Rebhühner, Fasanen. Terra-Verlag: 1-396. Budapest. 
24. Tarwid K., 1960: Szacowanie zbieżności nisz ekologicznych gatunków drogą oceny prawdopodobieństwa spotykania się ich w połowach. Ekol. pol., Ser. B 2: 115-130. Warszawa.

Received, July 28, 1966.

Institute of Ecology,

Polish Academy of Sciences,

Warszawa, Nowy Swiat 72.

\section{Zygmunt PIELOWSKI}

\section{BADANIA NAD ZAJĄCEM SZARAKIEM. XII. ORGANIZACJA PRZESTRZENNA POPULACJI}

\section{Streszczenie}

Sposób rozmieszczenia $\mathrm{w}$ areale populacji osobników zróżnicowanych pod względcm fizycznym i psychicznym oraz możliwości i sposób przestrzennego wykorzystania tego areału posiada najprawdopodobniej poważny wpływ na dynamikę liczebności. Badania miały za cel poznanie niektórych elementów rozmieszczenia pızestrzennego populacji zająca szaraka $w$ terenie typu podmiejskiego, silnie zurbanizowanym i w związku z tym mało przydatnym dla pomyślnego jej rozwoju. Ponieważ tego typu tereny są lowiecko użytkowane, to problem utrzymywania na nich zwierząt lownych w takim zagęszczeniu, które by umożliwiło w ogóle prowadzenie gospodarki lowieckiej, posiada duże znaczenie.

Badania przeprowadzono w latach 1961-1965 w pobliżu Warszawy. Metodykę hadań oparto $w$ zasadzie o t. zw. taksację pasowa (liczenie zajęcy na przebiegającym przez teren badań pasie, obejmującym 10\%\% całości jego powierzchni (Tabela 1 , Ryc. 1).

W wyniku badań stwierdzono sezonową migrację zajęcy wewnątrz areału populacji, pomiędzy polami a lasem, obejmującą kilkadziesiąt procent osobników (Tabela 2, Ryc. 5). Badając rozmieszczenie zajęcy $w$ zależności od rodzaju upraw stwierdzono, iż występuje wyraźna wybiórczość na korzyść upraw dających możliwie dużą osłonę (Ryc. 6). Badania uwidocznily również wybiórczość zajęcy w stosunku do różnych wariantów środowiska polnego (Tabela 4, Ryc. 7). Stwierdzono dalej, że niezależnie od zróżnicowania areału populacji na różne warianty środowiska polnego, istnieje drobiazgowe zróżnicowanie terenu na wycinki o różnym stopniu przydatności dla zajęcy (Tabela 4, Ryc. 8). Osobniki populacji wykazują tendencję do skupiskowego rozmieszczania się w terenie (Tabela 6, Ryc. 11).

Wydaje się, że stwierdzone objawy organizacji przestrzennej populacji zająca są między innymi wynikiem ciągłej dążności do zmniejszania stopnia narażenia osobników na niebezpieczeństwa i zaniepokojenia, są wyrazem przystosowania się populacji do istniejącej sytuacji ekologicznej. 
Tabelle 4.
Anzzahl der angetroffenen Hasen (Individuer,y) auf den einzelnen Abschnitten des Taxationsstreifens. 